Discussion Paper No. 1043

\title{
AN ENTRY GAME WITH LEARNING AND MARKET COMPETITION
}

\author{
Chia-Hui Chen \\ Junichiro Ishida \\ Arijit Mukherjee
}

October 2018

The Institute of Social and Economic Research Osaka University

6-1 Mihogaoka, Ibaraki, Osaka 567-0047, Japan 


\title{
An Entry Game with Learning and Market Competition*
}

\author{
Chia-Hui Chen† Junichiro Ishida ${ }^{\ddagger}$ and Arijit Mukherjee ${ }^{\S}$
}

October 26, 2018

\begin{abstract}
This paper provides a dynamic game of market entry to illustrate entry dynamics in an uncertain market environment. Our model features both private learning about the market condition and market competition, which give rise to the first-mover and secondmover advantages in a unified framework. We characterize symmetric Markov perfect equilibria and identify a necessary and sufficient condition for the first-mover advantage to dominate, which elucidates when and under what conditions a firm becomes a pioneer, an early follower or a late entrant. We also derive equilibrium payoff bounds to show that pioneering entry is generally payoff-enhancing, even though it is driven by preemption motives, and discuss efficiency properties of entry dynamics.
\end{abstract}

JEL Classification Number: D82, D83, L13

Keywords: market entry, market competition, private learning, signaling, entry timing.

\footnotetext{
${ }^{*}$ We thank Makoto Hanazono, Hideshi Itoh, and seminar and conference participants at Korea University, Kyoto University, Academia Sinica, SAET 2018, Pan Pacific Conference in Economic Research, and Contract Theory Workshop (CTW) for helpful comments and suggestions. The first author acknowledges financial support from JSPS KAKENHI Grant-in-Aid (B) JP16H03615. The second author acknowledges financial support from JSPS KAKENHI Grant-in-Aid (S) JP15H05728, (A) JP20245031, (B) JP16H03615, and (C) JP24530196 as well as the program of the Joint Usage/Research Center for Behavioral Economics at ISER, Osaka University. All errors are our own.

${ }^{\dagger}$ Institute of Economic Reserach, Kyoto University. Email: chchen@kier.kyoto-u.ac.jp

${ }^{\ddagger}$ Institute of Social and Economic Research, Osaka University. Email: jishida@iser.osaka-u.ac.jp

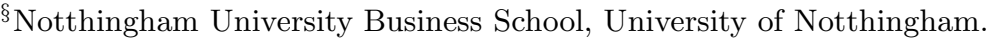

Email: arijit.mukherjee@nottingham.ac.uk
} 


\section{Introduction}

In the early 1990s, Boeing and Airbus independently began to investigate the potential profitability of the superjumbo market, i.e., the market for aircrafts with capacity for 500-1000 passengers. At the time, neither was sure whether the market would develop large enough to justify the huge investment required to enter this market. Specifically at issue was the uncertainty looming over the evolution of airline networks: if hub-and-spoke networks would remain dominant, it would favor bigger, though less cost efficient, aircrafts; if point-to-point networks would emerge instead, it would favor smaller medium-sized aircrafts. In the face of this uncertainty, the two companies eventually chose to follow divergent paths. In December 2000, Airbus formally announced to enter the market with commitment to developing and launching A380. In the meanwhile, Boeing came to believe that the fragmentation of the market due to the rise of point-to-point networks would limit demand for a superjumbo and decided to withdraw from the market.

This is the type of situation firms routinely encounter whenever there is a new (geographical) market, a new product, or a new technology becoming available; facing a new market opportunity, a potential entrant needs to conduct careful marketing research to gauge its profitability and decide whether and when to enter the market. The timing of market entry is in fact one of the most critical strategic decisions a firm must make, as is often emphasized in the fields of strategic management and marketing. ${ }^{1}$ Even as a single-firm decision problem, it is never straightforward to determine whether and when to enter the market when the eventual value of market entry is dissolved only gradually over time. The situation is even more compounded when there are multiple parties who interact and compete with each other, especially with information and payoff spillovers among them. In the superjumbo market, for instance, Airbus could have entered earlier, to which Boeing might have reacted differently, or Airbus's entry could have trigerred Boeing's subsequent entry, either immediately or after some additional marketing research. All these decisions and anticipated reactions are strategically linked and affect each other, possibly generating a plethora of dynamic entry patterns.

In this paper, we construct a dynamic game of market entry to capture this type of situation, with focus on a tradeoff faced by firms which contemplate to enter a market of unknown profitability. We consider an environment with two potential entrants, each of which independently decides whether and when to enter a new market. The profitability of the market is determined by the market condition (e.g., market size, production cost) which

\footnotetext{
${ }^{1}$ For instance, Lilien and Yoon (1990) note that "the choice of market-entry time is one of the major reasons for new product success or failure."
} 
is not known to anyone initially. Each firm privately collects information about the market condition over time, and decides to enter when it becomes sufficiently confident about the market. As a key departure from the existing literature, we introduce into this setting the following two factors.

- Market competition: A firm's flow profit is decreasing in the number of firms in the market.

- Private (pre-entry) learning and signaling via market entry: Before entry, each firm privately collects information about the market over time. As a consequence, a firm's market entry serves as a signal of its private information.

These two factors are often regarded as the primary sources of early-mover and latemover advantages in the literature ${ }^{2}$ and generate the tradeoff of our focus: the first one, the presence of market competition, implies a benefit of acting first whereas the second one, private learning and signaling via market entry, implies a benefit of waiting and learning from the rival's action. Our model thus captures the first-mover and second-mover advantages in a unified framework. The overall benefit of becoming a "market pioneer" is essentially determined by the way this tradeoff resolves.

To see the workings of our model, it is important to observe that since each firm starts with a common prior and gains more information over time, the signaling effect of market entry is generally weaker at early stages of the game. As time passes, however, it eventually reaches a point where a firm's entry reveals so much information that the rival firm immediately follows suit, thereby entirely dissipating the first-mover advantage. Due to this force, the game is generally divided into two distinct phases, called preemption and waiting for clarity, where the game begins with the preemption phase and then later switches to the waiting phase. The strategic nature of the problem differs significantly between the two phases. In the preemption phase, as a firm's entry can push back the timing of the rival firm's entry by cutting its residual demand, each firm prefers to enter slightly earlier than the rival. In the waiting phase, on the other hand, there is no such gain, and each firm prefers to enter slightly later to learn from the rival. For expositional purposes, we say that pioneering entry occurs, or a market pioneer emerges, when a firm enters the market in the preemption phase.

\footnotetext{
${ }^{2}$ For instance, Lieberman and Montgomery (1988) raise preemption of rivals as one of the first-mover advantages but also emphasize that "late movers can gain an edge through resolution of market or technological uncertainty." Incidentally, these two factors also played a central role in the Boeing-Airbus example: the market for large jetliners is a typical duopoly where the two firms compete intensely for the global market share; given the high entry cost into each submarket, pre-entry marketing research is also an integral part of their business operations.
} 
Although we base our analysis on a relatively simple setup, a technical complication arises from the fact that each firm privately observes signals and at the same time also learns from the other's action (or inaction). As a consequence, the firms' beliefs evolve in a rather complicated manner depending on their entry strategies, which along with the presence of market competition can easily make the analysis intractable. One of the major contributions of our analysis is to devise an analytical framework that is tractable enough to deliver some key insights for when and how a market pioneer emerges in a market where both learning and market competition are crucial factors. Specifically, we characterize symmetric Markov perfect equilibria and derive a necessary and sufficient condition for pioneering entry to occur in this environment. When this condition is satisfied, the firms enter the market at some positive rate in the preemption phase until it reaches a "saturation point" where the signaling effect of market entry becomes strong enough just to offset the first-mover advantage. Market entry then ceases to occur past this point, with neither firm taking any action. After a while, though, an uninformed firm would eventually become confident enough and willing to enter the market even without the chance to earn the monopoly rent. The model thus exhibits a rich, on-and-off, dynamics of market entry where the firms gradually enter the market at early and late stages, with the period of no entry in between.

We also identify equilibrium payoff bounds and argue that pioneering entry is generally payoff-enhancing for the firms even though it is driven by preemption motives. To see this result, note that the impact of pioneering entry on each firm's ex ante expected payoff is not entirely positive because the presence of market competition induces the firms to enter prematurely, compared to the optimal timing, in order to preempt the rival firm. Despite this efficiency loss, pioneering entry can still be socially beneficial as it reveals valuable information and inevitably facilitates the rival firm's learning. We show that due to this positive information spillover, pioneering entry is generally payoff-enhancing in that each firm's expected payoff is higher in the equilibrium with pioneering entry than the one without.

Finally, as noted earlier, the timing and order of market entry are a topic of utmost concern in the strategic management and marketing literature. ${ }^{3}$ The literature often classifies entry timing into three broad categories: pioneers, early followers, and late entrants. Our model generates a range of entry patterns consistent with this classification and hence contributes to this literature by clarifying when and under what conditions a firm becomes a pioneer, an early follower or a late entrant. We also examine the optimal timing of entry that maximizes the joint profit of the firms and find that the welfare impact of an entry pattern depends crucially

\footnotetext{
${ }^{3}$ Since the seminal work of Lieberman and Montgomery (1988), there is now a voluminous literature examining, mainly empirically, the extent of the first-mover advantages which is also known as the order-ofentry effect.
} 
on the timing of the first entry. Drawing on this fact, we argue, somewhat paradoxically, that the firms enter the market too early when there is a late entrant while they enter too late when there is an early follower.

\subsection{Related literature}

The major contribution of our analysis is that it incorporates market competition and private learning (and hence signaling via market entry) in an analytically tractable manner to deliver a comprehensive description of when and under what conditions a market pioneer emerges in equilibrium. Despite the fact that these two factors are often regarded as the primary sources of early-mover and late-mover advantages (Lieberman and Montgomery, 1988), they have been investigated rather independently and separately from one another in the existing literature: there are many related works which incorporate either one of these elements in various ways but very few which combine them in a unified framework.

First, our paper is related to works which examine the optimal timing of entry or exit with information externalities among the players. To name some, Chamley and Gale (1994) consider a model of strategic investment in which there are $N$ players, a random number $n$ of whom have an investment option. Assuming that the value of investment depends positively on the random number $n$, there is an incentive to wait and see others' investment decisions. Grenadier (1999) analyzes an option exercise game in which each player is endowed with private information about the true value of the option and hence the timing of exercise becomes a signal for other players. Murto and Välimäki (2011) analyze an exit game with private learning where each player receives a signal in each period which partially reveals his own type. A common thread of those works is that they do not consider the element of market competition, or negative payoff externalities, ${ }^{4}$ and focus more on issues such as investment delays and waves.

In contrast, models of market entry in industrial organization generally focus on market competition but not on dynamic learning. ${ }^{5}$ For instance, Levin and Peck (2003) analyze a duopoly model of market entry in which each firm privately observes its entry cost at the outset of the game. The market environment is similar to ours in that the first mover can

\footnotetext{
${ }^{4}$ In Chamley and Gale (1994), the value of investment increases as more players invest, which is a form of positive payoff externality. Genadier (1999) also extends the analysis to incorporate positive payoff externalities, e.g., due to network effects.

${ }^{5}$ Profit uncertainty also plays an eminent role in the context of foreign direct investment. Horstmann and Markusen $(1996,2015)$ consider a model in which a producer (the MNE) is unsure of the potential customer size and chooses either to contract with a local sales agent or to establish an owned local sales operation. While contracting with the local sales agent, the producer gains information about the customer size and switches to an owned sales operation if this option is found to be profitable. In their models, however, there is only one producer and hence no market competition.
} 
earn monopoly rents until the second mover arrives. Aside from the fact that there is no learning, a crucial difference is that the cost uncertainty in their model is individual-specific and hence a firm's entry does not reveal any useful information to the other firm. Rasmusen and Yoon (2012) analyze a duopoly model of market entry which incorporate both market competition and signaling. They consider a two-period model in which one of the firms is better informed about the market size than the other, and market entry by the informed firm hence becomes a signal of its private information. As in Levin and Peck (2003), however, the information structure is exogenously fixed at the outset of the game, which rules out the possibility of learning over time.

Bloch et al. (2015) consider a similar environment to ours but with a different information structure. They analyze a duopoly model of market entry where the firms face uncertainty regarding the common entry cost. ${ }^{6}$ In their model, as in our setting, the firms receive private signals about the entry cost, and the flow payoff of entry is decreasing in the number of firms in the market. The key difference from our setting is that the learning process in their model is "all or nothing" where a firm can perfectly identify the true state of nature with some probability in each period or learn nothing at all. This structure makes the belief system essentially binary: at any point in time, a firm is either fully informed about the state or has no additional information to the prior. They then exploit this structure and identify various kinds of equilibrium by letting the two types - informed and uninformed - coordinate their entry timing; as such, entry by the uninformed type is generally lumpy and occurs at some time with strictly positive probability. In contrast, the learning process in our model is gradual and continuous where each firm builds up confidence slowly over time, so that we need to keep track of the firms' beliefs which evolve in a rather complicated manner depending on their entry strategies. This difference requires us to adopt a different analytical approach and leads to different equilibrium properties.

A work that is more closely related to ours is a duopoly model of investment by Decamps and Mariotti (2004). ${ }^{7}$ As in our model, they assume that the quality of the project, which is common to both players, is not known ex ante and gradually revealed over time via the arrival of a bad (public) signal. In addition to this public learning, they further assume that once the leader has made investment, an additional signal is generated that may be used by the follower to adjust his investment decision. Although they focus mainly on the case with

\footnotetext{
${ }^{6}$ Bloch et al. (2014) also consider a similar setup but with firm-specific entry costs.

${ }^{7}$ The optimal timing of investment under uncertainty is investigated actively in the real options literature. Although much of the literature focuses on a single-agent decision problem, there are some attempts to incorporate strategic interactions into the real options approach (Trigeorgis, 1991; Grenadier, 1996; Weeds, 2002; Shackleton et al., 2004; Pawlina and Kort, 2006). This strand of literature generally assumes public learning with no information asymmetry among the competing players as in Decamps and Mariotti (2004).
} 
no market competition, they later extend the analysis to discuss the case where the follower's payoff is discounted by some fraction. In their model, however, learning is public, and there is hence no possibility of signaling.

\section{Model}

We consider a dynamic game of market entry where two potential entrants, labeled as firms 1 and 2, contemplate to enter the market of unknown profitability. The market condition is either good or bad, and each firm collects information before it makes an irreversible entry decision. There are two sources of information in this model: on one hand, each firm may observe a signal of the market condition which arrives stochastically over time; on the other hand, the entry decision of each firm is also publicly observable and hence serves as an additional signal. The fact that a firm can observe the rival's entry implies a benefit of "waiting," giving rise to the second-mover advantage. The tradeoff arises, however, as the profitability of each firm depends negatively on the number of firms in the market, meaning that the first one to enter the market can temporarily monopolize the market (until the second one arrives).

Time is continuous and extends from zero to infinity, and each firm decides whether to enter the market at discrete points in time $0, \Delta, 2 \Delta, \ldots$ by incurring the entry cost $c>0$. Throughout the analysis, we will focus on the continuous-time limit where the length of a time interval $\Delta$ shrinks to zero. As we will see below, once a firm enters, it is weakly optimal to stay in the market indefinitely, so that the firm has no further decision to make. We assume that each firm can observe the other firm's entry decisions but not the realized payoffs (or, equivalently, the market condition). ${ }^{8}$

Definition 1 A firm is called active if it has entered the market and inactive otherwise.

The market condition, which is common to both firms, is either good or bad. The prior probability of the market being good is $p_{0}$. The market condition is initially not known to either firm and is revealed only gradually over time. More precisely, a firm may privately observe a signal of the market condition as long as it remains inactive. The signal is of the bad-news type and arrives only if the market is bad: conditional on the market being bad, for an interval $[t, t+d t)$, an inactive firm observes a signal with probability $\lambda d t$. This specification means that the arrival of a signal indicates that the market condition is bad for

\footnotetext{
${ }^{8}$ This is essentially to assume that the profits are often realized with a substantial lag. In the superjumbo market, for instance, it easily took a decade, if not more, to see the eventual size of the market.
} 
sure. $^{9}$

Definition 2 An inactive firm is called informative if it has observed a signal and uninformative otherwise.

According to these definitions, we can classify each firm into three distinct categories: active, informed, and uninformed. In particular, when we refer to a firm as either informed or uninformed, it implies that it is inactive at the moment. As will become clear below, there are no further decisions to make if a firm is either active or informed, so that we generally focus on the problem of a firm that is currently uninformed.

The net profit a firm can earn is determined by the market condition and the number of firms in the market. If the market is good, an active firm earns $(\pi+\rho) \Delta$ per period if it is the only firm in the market and $\pi \Delta$ if both of them are in the market. We call $\rho$ the monopoly premium, which could depend on the extent of market competition, and in general assume $\rho>0$. If the market is bad, on the other hand, an active firm invariably earns zero profit. The net profit for an inactive firm is also normalized at zero, implying that there is no incentive to exit from the market ex post.

\section{Market entry under private learning}

\subsection{Belief formation}

From the viewpoint of an uninformed firm, there are three possible states as we describe below:

1. The market is good (state $G$ );

2. The market is bad, and the other firm is uninformed (state $B U$ );

3. The market is bad, and the other firm is informed (state $B I$ ).

An uninformed firm's belief is hence defined in two dimensions and denoted as $\left(p_{t}, q_{t}\right)$ where: (i) $p_{t}$ is the conditional probability that the market is good (state $G$ ); (ii) $q_{t}$ is the conditional probability that the market is bad and the other firm is uninformed (state $B U$ ). By definition, $1-p_{t}-q_{t}$ is the conditional probability that the market is bad and the other firm is informed (state $B I$ ). All the probabilities are conditional on the firm being inactive and uninformed. In what follows, we focus on symmetric Markov perfect Bayesian equilibria with the belief $\left(p_{t}, q_{t}\right)$ as the state variable.

\footnotetext{
${ }^{9}$ We make this assumption purely for tractability. An important implication of this assumption is that a firm's belief gradually improves over time as long as it stays uninformed, which is essential to make pre-entry learning meaningful. Our results should hold in a qualitative sense even if we consider a more complicated information process as long as this structure is retained.
} 


\subsection{Second mover}

It is quite straightforward to derive the optimal strategy for the second mover who, with no strategic concerns, simply enters if the current belief $p_{t}$ is high enough. Observe that the belief $p_{t}$ increases monotonically over time as long as the firm observes no signal.

Given some belief $p$, the expected joint profit of entering now is given by

$$
p \frac{\pi}{r}-c
$$

which increases over time. Alternatively, the firm may wait and enter at the next instant upon observing no signal. Since the firm observes no signal with probability $1-(1-p) \lambda \Delta$, the expected profit of this waiting strategy is given by

$$
(1-r \Delta)\left(p \frac{\pi}{r}-c+(1-p) \lambda \Delta c\right)
$$

The cost of adopting this strategy is obviously the foregone profit. On the other hand, by waiting, the firm can collect more information, the benefit of which is captured by $(1-p) \lambda \Delta c$. It is clear that the benefit of avoiding wrong entry is decreasing in the current belief.

It follows from these that the firm enters the market now if and only if

$$
p \frac{\pi}{r}-c \geq(1-r \Delta)\left(p \frac{\pi}{r}-(p+(1-p)(1-\lambda \Delta)) c\right),
$$

which is further simplified to

$$
r \Delta\left(p \frac{\pi}{r}-c\right) \geq(1-r \Delta)(1-p) c \lambda \Delta .
$$

This condition gives the cutoff belief $\bar{p}$ which converges in the limit to

$$
\bar{p}=\frac{(r+\lambda) c}{\pi+\lambda c}
$$

suggesting that the second mover enters the market once and for all when the belief $p_{t}$ reaches the threshold $\bar{p}$. We assume that this threshold is smaller than one, so that a firm enters if it knows that the market condition is good almost surely.

Assumption $1 \bar{p}<1 \Leftrightarrow \frac{\pi}{r}>c$.

Now suppose that the first mover enters the market at some time $t$. As this necessarily implies that the first mover has observed no signal, the second mover's belief makes a discrete jump after the entry. The updated belief is obtained as

$$
\lim _{\Delta \rightarrow 0} p_{t+\Delta}=\phi_{t}:=\frac{p_{t}}{p_{t}+q_{t}}
$$


which indicates the amount of information revealed by a market entry. Note in particular that no firm is informed at time 0, i.e., $q_{0}=1-p_{0}$ and $p_{0}=\phi_{0}$, meaning that a firm's entry reveals no additional information.

If the updated belief exceeds $\bar{p}$, the firm will follow immediately at the next instant, so that the first mover can appropriate almost no monopoly rent in the limit.

Lemma 1 An uninformed firm follows immediately after observing the other firm's entry at $t$ if and only if $\phi_{t} \geq \bar{p}:=\frac{(\lambda+r) c}{\pi+\lambda c}$.

\subsection{First mover}

We now turn to the first mover's problem which is far more complicated than the second mover's problem described above. Throughout the analysis, we often denote by $(p, q)$ the current belief and by $\left(p^{\prime}, q^{\prime}\right)$ the belief at the next instant when no entry will have occurred. Let $\sigma$ be the (symmetric Markov) behavior strategy where $\sigma(p, q) \Delta$ is the probability of market entry in a time period, conditional on the firm being uninformed (and both firms being inactive) when the current belief is $(p, q)$. In what follows, we often write $s=\sigma(p, q)$ and $s^{\prime}=\sigma\left(p^{\prime}, q^{\prime}\right)$ to save notation.

As mentioned, the major technical complication of our analysis arises from the fact that the evolution of the belief during this phase depends on the first mover's strategy $\sigma$. More precisely, in states $G$ and $B U$, the rival firm enters the market with probability $s \Delta$; in state $B I$, knowing that the market is bad, the rival firm never enters. Moreover, in states $B U$ and $B I$, the firm observes no signal and remains uninformed with probability $1-e^{-\lambda \Delta}$. Finally, the state changes from $B U$ to $B I$ when the rival firm observes a signal, which occurs with probability $1-e^{-\lambda \Delta}$. Given the current belief $(p, q)$ and $s=\sigma(p, q)$, the next-period belief $\left(p^{\prime}, q^{\prime}\right)$ is hence computed as

$$
\begin{aligned}
& p^{\prime}=\frac{(1-s \Delta) p}{(1-s \Delta)\left(p+q e^{-\lambda \Delta}\right)+(1-p-q) e^{-\lambda \Delta}}, \\
& q^{\prime}=\frac{(1-s \Delta) e^{-2 \lambda \Delta} q}{(1-s \Delta)\left(p+q e^{-\lambda \Delta}\right)+(1-p-q) e^{-\lambda \Delta}},
\end{aligned}
$$

with the initial prior given by $q_{0}=1-p_{0}$.

The current state of the economy is thoroughly characterized by $(p, q)$. What is particularly crucial is $\phi:=\frac{p}{p+q}$ which essentially determines the amount of information revealed by an entry. Fortunately, while $(p, q)$ may follow a quite complicated path, it is relatively straightforward to compute $\phi$ as it is independent of the first-mover strategy $\sigma$; from (1) and 
(2), we obtain

$$
\phi^{\prime}:=\frac{p^{\prime}}{p^{\prime}+q^{\prime}}=\frac{p}{p+q e^{-2 \lambda \Delta}}>\phi,
$$

which indicates that $\phi_{t}$ monotonically increases over time for any given strategy $\sigma$.

\subsubsection{The waiting phase: $\phi \geq \bar{p}$}

To characterize the first mover's optimal strategy, there are two cases we need to consider, depending on whether $\phi$ exceeds $\bar{p}$ or not. We start with the case where the current belief $(p, q)$ is such that $\phi \geq \bar{p}$, so that an uninformed firm immediately follows the rival firm. Note that this is the "winner's curse range" where the first mover can monopolize the market only if the market condition is bad. As this is a phase where the second-mover advantage dominates, we call it the waiting phase.

We essentially follow the same procedure to derive the continuation equilibrium in this phase, by comparing the expected payoffs of entering now and at the next instant. Suppose first that firm 1 chooses to enter now. Conditional on the market being good, firm 2 stays inactive with probability $1-s \Delta$, which allows firm 1 to monopolize the market for a period. As such, since firm 1 earns $(\pi+(1-s \Delta) \rho) \Delta$ in the first period after entry and $\pi \Delta$ thereafter, the expected profit of entering at $t$ is

$$
p\left(\frac{\pi}{r}+(1-s \Delta) \rho \Delta\right)-c
$$

Now consider an alternative strategy in which firm 1 waits and enters at the next instant if it is still uninformed. In the meanwhile, firm 2 may or may not enter at $t$. There are two possibilities. First, firm 2 enters now and firm 1 follows at the next instant, which occurs with probability $s \Delta(p+q(1-\lambda \Delta))$. The expected payoff from this contingency is

$$
(1-r \Delta) s \Delta\left(p \frac{\pi}{r}-(p+q(1-\lambda \Delta)) c\right) .
$$

Second, firm 2 does not enter now but firm 1 enters at the next instant, which occurs with probability $(1-s \Delta)(p+q(1-\lambda \Delta))+(1-p-q)(1-\lambda \Delta)$. The expected payoff from this contingency is

$$
(1-r \Delta)\left((1-s \Delta) p\left(\frac{\pi}{r}+\left(1-s^{\prime} \Delta\right) \rho \Delta\right)-((1-s \Delta)(p+q(1-\lambda \Delta))+(1-p-q)(1-\lambda \Delta)) c\right),
$$

where $s^{\prime}=\sigma\left(p^{\prime}, q^{\prime}\right)$. From these, the expected payoff of this waiting strategy is obtained as

$$
(1-r \Delta)\left(p \frac{\pi}{r}-(p+(1-p)(1-\lambda \Delta)) c+(1-s \Delta)\left(1-s^{\prime} \Delta\right) p \rho \Delta\right)
$$


taking $s$ and $s^{\prime}$ as given. ${ }^{10}$

Comparing (3) and (4), the waiting strategy yields a lower payoff if

$$
r\left(p \frac{\pi}{r}-c\right)+(1-s \Delta) s^{\prime} \Delta p \rho>(1-p) \lambda c
$$

which shows the tradeoff between entering now and waiting for an instant. By entering now, firm 1 may monopolize the market and obtain an additional payoff of $(1-s \Delta) p s^{\prime} \Delta \rho$ for a time period. By waiting until the next instant, the firm may receive additional information and save $(1-p) \lambda c$ by avoiding wrong entry; however, a firm also suffers a loss $r\left(p \frac{\pi}{r}-c\right)$ since the continuation payoff is realized an instant later.

The following statement establishes that the firms gradually enter at some positive rate once the belief reaches the threshold $\bar{p}$. It is relatively straightforward to verify that this constitutes an equilibrium. The proposition further suggests that this is the unique symmetric continuation equilibrium of the waiting phase.

Proposition 1 For $\phi_{t} \geq \bar{p}>p_{t}$, there exists a unique symmetric continuation equilibrium in which:

1. Neither firm enters until $p_{t}$ reaches $\bar{p}$;

2. When $p_{t}$ reaches $\bar{p}$, the two firms start entering at a rate to keep $p_{t}=\bar{p}$;

3. Once a firm enters, the other firm immediately follows at the next instant.

Proof. See Appendix.

If $p_{0}>\bar{p}$, we have a situation where $\phi_{0}>p_{0}>\bar{p}$. In this case, the firms enter with strictly positive probability at time 0 so that $\bar{p}>p_{\Delta}$ (see Lemma 5 in Appendix). Proposition 1 thus exhausts all the possibilities and completely characterizes the continuation equilibrium for any $\phi_{t} \geq \bar{p}$.

\subsubsection{The preemption phase: $\bar{p}>\phi$}

The strategic nature of the problem flips once $\phi$ gets below this threshold $\bar{p}$. As entry in this phase is driven by the first-mover advantage and preemption motives, we call it the

\footnotetext{
${ }^{10}$ Note that this is the expected payoff when firm 1 enters at the next instant regardless of whether firm 2 enters now or not, which may not hold if $\lim _{\Delta \rightarrow 0} s \Delta>0$. For instance, if $\lim _{\Delta \rightarrow 0} s \Delta=1$ and firm 2 does not enter, firm 1 knows for sure that the market is bad, making it optimal never to enter. As we will see below, however, the firms always enter smoothly in the waiting phase, so that this condition is always valid.
} 
preemption phase. Let $\tau^{*}$ denote the time of the earliest possible entry in equilibrium, which is define as

$$
\tau^{*}:=\inf \left\{t: \sigma_{t}>0\right\}
$$

For exposition, we say that pioneering entry occurs, or a market pioneer emerges, if $s>0$ for any $(p, q)$ such that $\bar{p}>\phi$ or, equivalently, $\bar{p}>\phi_{\tau^{*}}{ }^{11}$ We call it "pioneering" because, as we will see later, it generates valuable information and is generally beneficial to the rival firm, despite the fact that it is driven by preemption motives.

Given $\bar{p}>\phi$, if a firm enters now, the rival firm's belief jumps up but is still lower than $\bar{p}$. The second mover thus will not enter immediately, and the first mover can monopolize the market for some duration of time which we denote by $\delta_{t}$. Since the second mover waits until the belief reaches $\bar{p}, \delta_{t}$ is computed as

$$
\delta_{t}=\frac{1}{\lambda} \ln \frac{\bar{p} q_{t}}{p_{t}(1-\bar{p})}
$$

Note that for a given $p_{0}, \phi_{t}$ depends only on $t$, and so is $\delta_{t}$. The expected payoff of entering now is then given by

$$
p \frac{\pi+\left(1-e^{-r \delta_{t}}\right) \rho}{r}-c
$$

The equilibrium allocation becomes much more complicated when pioneering entry actually occurs on the equilibrium path with positive probability. We can nonetheless establish that any (symmetric) equilibrium must in general take the following form.

Proposition 2 Suppose that pioneering entry occurs in equilibrium with some positive probability. Then, in any symmetric equilibrium, there exist $\underline{\tau}$ and $\bar{\tau} \in(\underline{\tau}, \infty)$ such that

$$
s_{t} \begin{cases}\in(0, \infty) & \text { for } t \in\left(\tau^{*}, \underline{\tau}\right) \cup(\bar{\tau}, \infty), \\ =0 & \text { for } t \in(\underline{\tau}, \bar{\tau}) .\end{cases}
$$

Moreover, $\phi_{\underline{\tau}}<\bar{p}$.

Proof. See Appendix.

In equilibrium, the firms enter smoothly over time except at the outset of the game. ${ }^{12}$ The proposition suggests that if pioneering entry ever occurs, there is an interval during

\footnotetext{
${ }^{11}$ Note that our definition of market pioneer differs from the conventional one: in the literature, it simply refers to the first entrant in a new market (Robinson and Fornell, 1985).

${ }^{12}$ At time 0 , the firms may enter with some strictly positive probability.
} 
which the firms enter at some positive rate, followed by an interval of no entry $(\underline{\tau}, \bar{\tau})$. This is because, although the firms have less private information and face more uncertainty early on, the fact that they have less information means that a firm's entry reveals less information, thereby making the preemption effect stronger. When this first-mover advantage dominates the cost of entering prematurely with insufficient information, the firms enter with some positive probability in the preemption phase.

\section{Equilibrium}

\subsection{The constrained problem}

To analyze the possibility of pioneering entry, we consider a hypothetical situation in which a firm, say firm 2, can enter only after the other firm, i.e., firm 1, enters. As it turns out, this constrained version of the problem, which excludes the possibility of entry competition, provides enough information to see when pioneering entry occurs in the original problem.

Under the restriction that firm 2 must be the second mover, the problem faced by firm 1 is substantially simpler: firm 1 simply decides when to enter conditional on having observed no signal. Provided that firm 2 never enters, firm 1's belief pair at $t$ is given by

$$
p_{t}=\frac{p_{0}}{p_{0}+\left(1-p_{0}\right) e^{-\lambda t}}, q_{t}=\frac{\left(1-p_{0}\right) e^{-2 \lambda t}}{p_{0}+\left(1-p_{0}\right) e^{-\lambda t}},
$$

which depends only on $t$. As a consequence, the expected payoff of entering at $t$ can also be written as a function of $t$. Let $\hat{\Pi}_{s}(t)$ denote the expected payoff of entering at $t$, evaluated at time $s$, under the restriction that firm 2 must be the second mover. If firm 1 enters at time $t$, firm 2 will wait until $p_{t}$ reaches $\bar{p}$. Evaluated at time 0 , we obtain

$$
\hat{\Pi}_{0}(t)=e^{-r t}\left(p_{0} \frac{\pi+\left(1-e^{-r \delta_{t}}\right) \rho}{r}-\left(p_{0}+\left(1-p_{0}\right) e^{-\lambda t}\right) c\right)
$$

If $\phi_{t} \geq \bar{p}$, then $\delta_{t}=\Delta$ which converges to zero in the limit. In contrast, if $\bar{p}>\phi_{t}$, firm 2 must wait to collect more information, giving firm 1 some time to monopolize the market. The incentive for pioneering entry thus hinges crucially on $\delta_{t}$.

Suppose first that there is no pioneering entry and $\delta_{t}=0$. Along with the fact that $\phi_{t}$ is monotonically increasing, the continuation equilibrium characterized in Lemma 1 suggests that the earliest possible entry occurs when the belief reaches $\bar{p}$. Define $\tau^{\mathrm{NP}}$ such that

$$
e^{-\lambda \tau^{\mathrm{NP}}}=\frac{p_{0}(1-\bar{p})}{\bar{p}\left(1-p_{0}\right)}=\frac{p_{0}(\pi-r c)}{\left(1-p_{0}\right)(\lambda+r) c}
$$

so that given $p_{0}$, the earliest possible entry occurs at $\tau^{\mathrm{NP}}$. From (6), we obtain

$$
\delta_{t}=\frac{1}{\lambda} \ln \frac{\bar{p} q_{t}}{p_{t}(1-\bar{p})}=\frac{1}{\lambda} \ln \frac{\bar{p}\left(1-p_{0}\right) e^{-2 \lambda t}}{p_{0}(1-\bar{p})}=\tau^{\mathrm{NP}}-2 t,
$$


meaning that $\delta_{t}>0$ if and only if $\frac{\tau^{\mathrm{NP}}}{2}>t$. Let $\Pi^{\mathrm{NP}}:=\max _{t \in\left(\frac{\tau^{\mathrm{NP}}}{2}, \infty\right)} \hat{\Pi}_{0}(t)$ denote the expected payoff without pioneering entry, which can be written as

$$
\Pi^{\mathrm{NP}}=\hat{\Pi}_{0}\left(\tau^{\mathrm{NP}}\right)=e^{-r \tau^{\mathrm{NP}}}\left(p_{0} \frac{\pi}{r}-\left(p_{0}+\left(1-p_{0}\right) e^{-\lambda \tau^{\mathrm{NP}}}\right) c\right) .
$$

Now suppose that firm 1 enters early when $\delta_{t}$ is still positive or alternatively $\frac{\tau^{\mathrm{NP}}}{2}>t$. If firm 1 enters at $t$, firm 2 's belief jumps up to $\phi_{t}$, but firm 2 still needs to wait until the belief reaches $\bar{p}$, which allows firm 1 to monopolize the market for some time $\delta_{t}$. Therefore, the expected payoff of entering at $t \in\left[0, \frac{\tau^{\mathrm{NP}}}{2}\right]$ is given by

$$
\hat{\Pi}_{0}(t)=e^{-r t}\left(p_{0} \frac{\pi+\left(1-e^{-r\left(\tau^{\mathrm{NP}}-2 t\right)}\right) \rho}{r}-\left(p_{0}+\left(1-p_{0}\right) e^{-\lambda t}\right) c\right) .
$$

Note that this problem is well defined only if $\tau^{\mathrm{NP}}>0$. In what follows, therefore, we assume that this is indeed the case.

Assumption $2 \tau^{\mathrm{NP}}>0 \Leftrightarrow \bar{p}>p_{0}$.

The optimal timing of entry under the entry restriction can be found by maximizing $\hat{\Pi}_{0}$, which is a strictly concave function of $t$ as we show below.

Lemma 2 In the limit, there exits a unique $\tau^{\mathrm{P}} \in\left[0, \frac{\tau^{\mathrm{NP}}}{2}\right]$ which maximizes $\hat{\Pi}_{0}(t)$. Letting

$$
\mu(t):=-p_{0}(\pi+\rho-r c)-p_{0} \rho e^{-r\left(\tau^{\mathrm{NP}}-2 t\right)}+\left(1-p_{0}\right)(\lambda+r) c e^{-\lambda t},
$$

the optimal timing of pioneering entry, denoted by $\tau^{\mathrm{P}}$, is given by

$$
\tau^{\mathrm{P}}= \begin{cases}0 & \text { if } 0 \geq \mu(0), \\ \hat{\tau} & \text { if } \mu(0)>0>\mu\left(\frac{\tau^{\mathrm{NP}}}{2}\right), \\ \frac{\tau^{\mathrm{NP}}}{2} & \text { if } \mu\left(\frac{\tau^{\mathrm{NP}}}{2}\right) \geq 0\end{cases}
$$

where $\hat{\tau}$ solves $\mu(\hat{\tau})=0$.

Proof. See Appendix.

Let $\Pi^{\mathrm{P}}:=\max _{t \in\left[0, \frac{\tau^{\mathrm{NP}}}{2}\right]} \hat{\Pi}_{0}(t)$ denote the expected payoff under the restriction that firm 2 must be the second mover, which can be written as

$$
\Pi^{\mathrm{P}}=\hat{\Pi}_{0}\left(\tau^{\mathrm{P}}\right)=e^{-r \tau^{\mathrm{P}}}\left(p_{0} \frac{\pi+\left(1-e^{-r\left(\tau^{\mathrm{NP}}-2 \tau^{\mathrm{P}}\right)}\right) \rho}{r}-\left(p_{0}+\left(1-p_{0}\right) e^{-\lambda \tau^{\mathrm{P}}}\right) c\right) .
$$

As the monopoly premium becomes larger, it becomes more costly to wait and collect more information. As a consequence, the optimal timing of pioneering entry moves forward with an increase in the monopoly premium. 


\subsection{The emergence of a market pioneer}

Under the restriction that firm 2 must be the second mover, it is optimal for firm 1 to enter once and for all at $\tau^{\mathrm{P}}$ if $\Pi^{\mathrm{P}}>\Pi^{\mathrm{NP}}$. Clearly, though, this does not constitute an equilibrium when firm 2 is also an active player who can enter the market at any point in time: if firm 2 knows that firm 1 enters at $\tau^{\mathrm{P}}$ with certainty, there is always an incentive for firm 2 to enter slightly earlier at $\tau^{\mathrm{P}}-\Delta$ which allows it to monopolize the market for a while. As this argument and Proposition 2 indicate, the firms must adopt mixed strategies when they compete to be the first mover. Even then, these payoffs are still useful as they provide a necessary and sufficient condition for a market pioneer to emerge in equilibrium.

Proposition 3 Pioneering entry occurs in equilibrium if and only if $\Pi^{\mathrm{P}}>\Pi^{\mathrm{NP}}$.

Proof. See Appendix.

The intuition behind Proposition 3 is quite simple. To see this, observe that in the equilibrium with no pioneering entry, each firm waits until time $\tau^{\mathrm{NP}}$ and then gradually enters past that point; the expected payoff in this equilibrium is hence $\Pi^{\mathrm{NP}}$ as in the constrained problem. Given this, the sufficiency is obvious, because if $\Pi^{\mathrm{P}}>\Pi^{\mathrm{NP}}$, a firm must have an incentive to deviate and enter with some positive probability in the preemption phase. On the other hand, the necessity comes from the fact that the entry competition can only lower the benefit of pioneering entry while raising the benefit of waiting due to the signaling effect (see the next section for more detail). As such, if there is no incentive to enter in the preemption phase in the constrained problem, then there is certainly no incentive to do so in the original problem.

Proposition 3 implies that as $\Pi^{\mathrm{P}}-\Pi^{\mathrm{NP}}$ becomes larger, the first-mover advantage becomes more salient, rendering pioneering entry more likely. It is hence important to clarify factors which affect $\Pi^{\mathrm{P}}-\Pi^{\mathrm{NP}}$. The following proposition clarifies under what conditions a market pioneer is more likely to emerge, which offers crucial welfare and policy implications.

Proposition 4 There exist $\hat{\rho}$ and $\hat{p}_{0} \in(0, \bar{p})$ such that pioneering entry occurs if and only if $\rho \in(\hat{\rho}, \infty)$ or $p_{0} \in\left(\hat{p}_{0}, \bar{p}\right)$.

Proof. See Appendix.

An important determinant of the value of pioneering entry is obviously the monopoly premium $\rho$, where an increase in $\rho$ facilitates pioneering entry. This is illustrated in figures 1 and 2 which depict $\hat{\Pi}_{0}(t)$ for different values of $\pi$ and $\rho$ (with $\pi+\rho$ fixed at one). 
[Figures 1 and 2 about here]

\subsection{Equilibrium payoff bounds}

Let $\Pi^{*}$ denote the expected equilibrium payoff for each firm where

$$
\Pi^{*}=e^{-r \tau^{*}}\left(p_{0} \frac{\pi+\left(1-e^{-r \delta_{\tau^{*}}}\right) \rho}{r}-\left(p_{0}+\left(1-p_{0}\right) e^{-\lambda \tau^{*}}\right) c\right) .
$$

If $\Pi^{\mathrm{NP}}>\Pi^{\mathrm{P}}$, the earliest possible entry occurs at time $\tau^{\mathrm{NP}}$, and $\Pi^{*}=\Pi^{\mathrm{NP}}$ as we have seen. If $\Pi^{\mathrm{P}}>\Pi^{\mathrm{NP}}$, on the other hand, there is a clear gain from becoming the first mover. We in general have $\Pi^{*}<\Pi^{\mathrm{P}}$ because the entry competition shifts the timing of entry forward, inducing the firms to start entering before the optimal time $\tau^{\mathrm{P}}$. The following result characterizes the timing of pioneering entry, provided that $\Pi^{\mathrm{P}}>\Pi^{\mathrm{NP}}$.

Proposition 5 Suppose that $\tau^{\mathrm{P}}>0$. If pioneering entry occurs in equilibrium, then $\frac{\tau^{\mathrm{NP}}}{2}>$ $\tau^{\mathrm{P}}>\tau^{*}$.

Proof. See Appendix.

The entry competition is self-defeating in that it induces the firms to enter before they collect enough information, thereby reducing their payoffs compared to the case where firm 2 can only follow firm 1 . The question is then whether this competition drives the value of the first-mover advantage down to zero. As it turns out, the firms' expected payoffs can actually improve when pioneering entry occurs with positive probability, because the second mover can benefit from the information revealed by the first mover's entry. The following result characterizes the equilibrium payoff bounds when $\Pi^{\mathrm{P}}>\Pi^{\mathrm{NP}}$.

Proposition 6 Suppose that $\Pi^{\mathrm{P}}>\Pi^{\mathrm{NP}}$ so that pioneering entry occurs with positive probability. Then, each firm's expected payoff falls in $\left(\Pi^{\mathrm{NP}}, \Pi^{\mathrm{P}}\right]$, i.e.,

$$
\Pi^{*}=e^{-r \tau^{*}}\left(p_{0} \frac{\pi+\left(1-e^{-r \delta_{\tau^{*}}}\right) \rho}{r}-\left(p_{0}+\left(1-p_{0}\right) e^{-\lambda \tau^{*}}\right) c\right) \in\left(\Pi^{\mathrm{NP}}, \Pi^{\mathrm{P}}\right]
$$

Proof. See Appendix.

To understand this result, especially why the expected payoff is not driven down to $\Pi^{\mathrm{NP}}$, it is important to understand the roles of the two types of externality that are present in this setting. On one hand, there is a negative payoff externality via market competition which is 
captured by $\rho$. The payoff externality is clearly the source of the entry competition. This is most clearly seen by supposing $\rho=0$, in which case

$$
\hat{\Pi}_{0}(t)=e^{-r t}\left(p_{0} \frac{\pi}{r}-\left(p_{0}+\left(1-p_{0}\right) e^{-\lambda t}\right) c\right),
$$

for all $t \in[0, \infty)$, suggesting that there is no preemption phase.

In contrast, as $\rho$ increases, the first-mover advantage becomes more salient, giving each firm an incentive to become a market pioneer. This entry competition forces the firms to enter earlier than the optimal timing $\tau^{\mathrm{P}}$, which necessarily lowers the expected payoff of becoming a market pioneer. In equilibrium, this expected payoff must be driven down to the expected payoff of becoming a follower which is strictly larger than $\Pi^{\mathrm{NP}}$ because of the information externality: with the first-mover's entry providing additional information, the follower can enter earlier than $\tau^{\mathrm{NP}}$ and hence on average achieve a higher payoff.

\section{$5 \quad$ Efficiency}

\subsection{The social planner's problem}

To derive efficiency properties of the model, we now consider a social planner who attempts to maximize the joint profit of the firms. Since the first-best allocation is rather trivial in this setting, ${ }^{13}$ here we focus on the second-best allocation where the social planner is subject to the same informational constraints as the firms. Specifically, we consider an environment in which the social planner specifies the entry timing $\left(\tau_{1}, \tau_{2}\right)$ such that firm $i$ can enter the market only at time $\tau_{i}{ }^{14}$ Without loss of generality, we assume $\tau_{1} \leq \tau_{2}$.

For a given pair $\left(\tau_{1}, \tau_{2}\right)$, the joint profit is obtained as

$$
\Pi\left(\tau_{1}, \tau_{2}\right)=e^{-r \tau_{1}}\left(p_{0} \frac{\pi+\rho}{r}-\left(p_{0}+\left(1-p_{0}\right) e^{-\lambda \tau_{1}}\right) c\right)+e^{-r \tau_{2}}\left(p_{0} \frac{\pi-\rho}{r}-\left(p_{0}+\left(1-p_{0}\right) e^{-\lambda\left(\tau_{1}+\tau_{2}\right)}\right) c\right) .
$$

Two remarks are in order regarding the two types of externality in this setting. First, firm 2's belief at $\tau_{2}$ is $\frac{p_{0}}{p_{0}+\left(1-p_{0}\right) e^{-\lambda\left(\tau_{1}+\tau_{2}\right)}}$, rather than $\frac{p_{0}}{p_{0}+\left(1-p_{0}\right) e^{-\lambda \tau_{2}}}$, because of the positive information externality of the first entry. Second, the second entry contributes only $\pi-\rho$ to the joint profit (while its private gain is $\pi$ ) due to the negative payoff externality, which corresponds to what is often referred to as the "business-stealing effect" in standard static oligopoly models.

\footnotetext{
${ }^{13}$ The first-best allocation may be defined as the one that maximizes the joint profit when the market condition is known to the social planner. Then, the problem is clearly trivial: if the market is good, the firms should enter immediately at time 0 ; if not, they should never enter.

${ }^{14}$ Given that each firm has only one chance to enter the market, it chooses to enter if and only if it is profitable to do so at the time.
} 
It is also important to note that because of the payoff externality, there may arise a case where it is socially optimal to have only one firm in the market. This is the case if

$$
c \geq \frac{\pi-\rho}{r}
$$

in which case the social planner would allow only one firm to enter the market $\left(t_{2}=\infty\right)$. Since this case is relatively straightforward, we restrict our attention to the case where it is socially optimal to have two firms whenever the market condition is good.

Assumption $3 \pi-\rho>r c$.

\subsection{The socially optimal timing of entry}

We begin with the optimal timing of the second entry. Given $\tau_{1}$, the social planner's problem is defined as

$$
\max _{\tau_{2}} e^{-r \tau_{2}}\left(p_{0} \frac{\pi-\rho}{r}-\left(p_{0}+\left(1-p_{0}\right) e^{-\lambda\left(\tau_{1}+\tau_{2}\right)}\right)\right) .
$$

The first-order condition is then given by

$$
p_{0}(\pi-\rho-r c)=\left(1-p_{0}\right)(\lambda+r) c e^{-\lambda\left(\tau_{1}+\tau_{2}\right)} .
$$

The left-hand side indicates the foregone benefit of delaying the second entry while the righthand side is the benefit of collecting more information. Let $\tau_{2}^{* *}\left(\tau_{1}\right)$ denote the socially optimal timing of the second entry as a function of $\tau_{1}$. If there exists an interior solution $\tau_{2}^{* *}\left(\tau_{1}\right)>\tau_{1}$, it must solve

$$
p_{\tau_{2}^{* *}}=\frac{p_{0}}{p_{0}+\left(1-p_{0}\right) e^{-\lambda\left(\tau_{1}+\tau_{2}^{* *}\left(\tau_{1}\right)\right)}}=\frac{(\lambda+r) c}{\pi-\rho+\lambda c}>\bar{p} .
$$

Define $T^{* *}=\tau_{1}+\tau_{2}^{* *}\left(\tau_{1}\right)$ for $\tau_{2}^{* *}\left(\tau_{1}\right)>\tau_{1}$. From (9), we obtain

$$
e^{-\lambda T^{* *}}=\frac{p_{0}(\pi-\rho-r c)}{\left(1-p_{0}\right)(\lambda+r) c}
$$

which depends only on the primitives of the model. This means that if firm 1 enters at time $t \in\left[0, \frac{T^{* *}}{2}\right)$, firm 2 waits until time $T^{* *}-t$ and enters if it is still uninformed at the time.

Letting $\Pi^{* *}\left(\tau_{1}\right):=\Pi\left(\tau_{1}, \tau_{2}^{* *}\left(\tau_{1}\right)\right)$, the joint profit for $\tau_{1} \in\left[0, \frac{T^{* *}}{2}\right)$ can be written as $\Pi^{* *}\left(\tau_{1}\right)=e^{-r \tau_{1}}\left(p_{0} \frac{\pi+\rho}{r}-\left(p_{0}+\left(1-p_{0}\right) e^{-\lambda \tau_{1}}\right) c\right)+e^{-r\left(T^{* *}-\tau_{1}\right)}\left(p_{0} \frac{\pi-\rho}{r}-\left(p_{0}+\left(1-p_{0}\right) e^{-\lambda T^{* *}}\right) c\right)$.

If firm 1 enters after time $\frac{T^{* *}}{2}$, on the other hand, firm 2 follows immediately after the first entry. In the limit, the joint profit for $\tau_{1} \in\left[\frac{T^{* *}}{2}, \infty\right)$ converges to

$\Pi^{* *}\left(\tau_{1}\right)=e^{-r \tau_{1}}\left(p_{0} \frac{\pi+\rho}{r}-\left(p_{0}+\left(1-p_{0}\right) e^{-\lambda \tau_{1}}\right) c\right)+e^{-r \tau_{1}}\left(p_{0} \frac{\pi-\rho}{r}-\left(p_{0}+\left(1-p_{0}\right) e^{-2 \lambda \tau_{1}}\right) c\right)$. 
For $\tau_{1} \in\left[0, \frac{T^{* *}}{2}\right)$, the first-order condition is obtained as

$$
p_{0}(\pi+\rho-r c)=\left(1-p_{0}\right)(\lambda+r) c e^{-\lambda \tau_{1}^{* *}}+\frac{\lambda}{\lambda+r} p_{0}(\pi-\rho-r c) e^{-r\left(T^{* *}-2 \tau_{1}^{* *}\right)} .
$$

Note that the second term of the right-hand side indicates the benefit of the information externality which is increasing in $\tau_{1} \cdot{ }^{15}$ For $\tau_{1} \in\left[\frac{T^{* *}}{2}, \infty\right)$, the first-order condition is obtained as

$$
2 p_{0}(\pi-r c)=\left(1-p_{0}\right)\left((\lambda+r)+(2 \lambda+r) e^{-\lambda \tau_{1}^{* *}}\right) c e^{-\lambda \tau_{1}^{* *}}
$$

As above, let $\tau_{1}^{* *}$ denote the socially optimal timing of the first entry.

Proposition 7 (i) $T^{* *}>\tau^{\mathrm{NP}}$. (ii) $\tau_{1}^{* *}>\tau^{\mathrm{P}}$ if $\tau^{\mathrm{P}}>0$. (iii) $\tau_{1}^{* *}$ may be larger or smaller than $\tau^{\mathrm{NP}}$ where $\tau^{\mathrm{NP}}>\tau_{1}^{* *}$ if

$$
\frac{\lambda+r}{2 \lambda+r}>\frac{p_{0}(\pi-r c)}{\left(1-p_{0}\right)(\lambda+r) c}
$$

Proof. See Appendix.

\subsection{Pioneers, early followers, and late entrants}

Since the seminal work of Lieberman and Montgomery (1988), there is now a voluminous literature, mainly in the fields of marketing and strategic management, which examines the effects of entry timing and order. ${ }^{16}$ The literature often classifies entry timing into three broad categories: pioneers, early followers, and late entrants (Robinson and Fornell, 1985, Lambkin, 1988). Below, we first interpret our equilibrium outcomes according to this classification and discuss efficiency properties for each possible class of outcomes, with focus on the two types of externality.

Observe first that for a given set of parameters, we can pin down an equilibrium with an associated distribution of entry time. Each equilibrium outcome is generally characterized by a pair of realized entry times $\left(t_{1}, t_{2}\right)$ where $t_{1}\left(t_{2}\right)$ denotes the timing of the first (second) entry $\left(t_{i}=\infty\right.$ in the case of no entry). Our model then generates four classes of entry dynamics that are observationally distinguishable.

1. No entry $\left(t_{1}=t_{2}=\infty\right)$ : Neither firm chooses to enter, and the market never materializes.

\footnotetext{
${ }^{15}$ Due to this effect, the joint profit may not be concave for $\tau_{1} \in\left[0, \frac{T^{*}}{2}\right)$.

${ }^{16}$ The literature generally focuses on whether there is any enduring advantage for early entrants. Although we do not consider this possibility, our model can be easily extended to incorporate this type of first-mover advantage.
} 
2. Only one entry $\left(t_{1}<t_{2}=\infty\right)$ : Only one firm enters while the other firm chooses not to follow. This is the case of premature entry (as in the Boeing-Airbus case).

3. Late entrant $\left(t_{1}<t_{2}<\infty\right)$ : A firm enters in the preemption phase and is followed by the other firm with some time lag. The two entries are spaced apart in time.

4. Early follower $\left(t_{1} \approx t_{2}<\infty\right)$ : A firm enters in the waiting phase and is immediately followed by the other firm. The two entries are clustered together.

The first two cases occur only when the market condition is bad, reflecting the obvious fact that no successful market can be monopolized forever. Welfare implications in these cases are rather straightforward, even without going through any detailed analysis. Given that the market condition is bad, the first case is (first-best) efficient as the firms correctly decide not to enter the market. In the second case, one of the firms fails to observe a signal in time and erroneously enters the market which is not sufficiently profitable. This case is clearly inefficient regardless of the exact timing of the sole entry.

The latter two cases admit two entrants and are often the focus of attention in the existing literature. Parts (i) and (ii) of Proposition 7 concern the case with a late entrant and suggest that the firms enter the market too early compared to the social optimum.

- Part (i) states that the equilibrium timing of the second entry is earlier than the socially optimal timing. ${ }^{17}$ This is due to the negative payoff externality: when the second entry occurs, the firm's average net payoff when the market is good is $\pi-r c$ while its contribution to the joint profit is only $\pi-\rho-r c$ due to the business-stealing effect. ${ }^{18}$ In the optimal allocation, therefore, the entry threshold is higher and the firm should wait longer to collect more information.

- Part (ii) states that the equilibrium timing of the first entry is also earlier than the socially optimal timing. This is due to the positive information externality: if the first entry occurs later, it reveals more information and benefits the other firm. The first entrant not only ignores this benefit of signaling $\left(\tau_{1}^{* *}>\tau^{\mathrm{P}}\right)$, but in equilibrium enters even earlier so as to reveal less information to the rival firm and delay its subsequent $\operatorname{entry}\left(\tau^{\mathrm{P}}>\tau^{*}\right)$.

\footnotetext{
${ }^{17}$ Given that the first entry occurs at some $t$, the second entry occurs at $\tau^{\mathrm{NP}}-t$ whereas the socially optimal timing is given by $T^{* *}-t$.

${ }^{18}$ The celebrated excess entry theorem (Mankiw and Winston, 1986; Suzumura and Kiyono, 1987; Lahiri and Ono, 1988) generally builds on this effect and demonstrates that the number of firms in a market can be too many in static oligopoly models. Our analysis complements this literature by extending this argument to a dynamic context, showing that market entry is too early with this same effect.
} 
In contrast, Part (iii) of the proposition concerns the case with an early follower. Although $\tau_{1}^{* *}$ can be larger or smaller than $\tau^{\mathrm{NP}}$, we argue that (12) is more likely to hold under plausible conditions, meaning that the firms enter too late in this contingency. ${ }^{19}$ The intuition behind this case is relatively clear. Once the game reaches the waiting phase, the clear winner is the one that becomes the follower as it can minimize the risk of wrong entry while losing almost no monopoly rent. This incentive to wait for the rival's action is often excessively strong, preventing the firms from entering the market at an opportune time.

Note that Proposition 7 implies a paradoxical fact which is worth emphasizing: the firms enter too early when there is a later entrant, and too late when there is an early follower. This is because the efficiency properties of entry dynamics are determined largely by the timing of the first entry which shapes the temporal distribution of market entry. The problem is that the timing of the first entry is hard to observe empirically in many cases, as the existence of a market is often not known to outside observers until its first entry actually occurs. Fortunately, though, the time lag between early and late entrants, is suggestive and provides enough information about the timing of the first entry. A general rule of thumb is that when market entry occurs successively in a short span of time (the case of early followers), chances are that the market was already ripe when the first entrant arrived, implying that the market opened up too late. In contrast, when market entry occurs sparsely over time (the case of late entrants), the first entrant is likely to be a true pioneer who entered when the market was still filled with uncertainty, even though the market opened up too early with substantial risk of premature entry.

\section{Conclusion}

In the existing literature, the roles of pre-entry learning and subsequent market competition have been investigated extensively but almost independently, and there are very few works which combine them in a unified framework. To fill this gap and provide a more comprehensive description of the tradeoff faced by potential market entrants, this paper constructs a dynamic model of market entry which features these two elements simultaneously. Primary applications of our analysis include new product markets, technology adoption and foreign direct investment among others.

As final note, we would like to make a brief remark about the possibility of asymmetric equilibria. Although we restrict our attention to symmetric equilibria throughout the analysis, our model also admits some asymmetric equilibria. We can indeed find at least one form

\footnotetext{
${ }^{19}$ Proposition 4 shows that if $p_{0}$ is small, (i) pioneering entry is less likely to occur, and (ii) (12) is more likely to hold. Therefore, if no market pioneer emerges in equilibrium, (12) is more likely to hold.
} 
of such equilibrium, as the strategies considered in the constrained problem constitute an (asymmetric) equilibrium when $\Pi^{\mathrm{NP}}-\Pi^{\mathrm{P}}$ is sufficiently large: if a firm can somehow commit to being the second mover, the optimal choice for the other firm is to enter once and for all at time $\tau^{\mathrm{NP}} .{ }^{20}$ We did not pursue this possibility in our analysis because asymmetric equilibria typically require a level of coordination that is somewhat unrealistic in the context of entry games. ${ }^{21}$ It is nonetheless of some interest, at least theoretically, to explore this possibility in future to obtain a more detailed characterization of asymmetric equilibria.

\section{References}

Bloch, F., Fabrizi, S. and Lippert, S., 2014, Learning, Entry and Competition with Uncertain Common Entry Costs, mimeo.

Bloch, F., Fabrizi, S. and Lippert, S., 2015, Learning and Collusion in New Markets with Uncertain Entry Costs, Economic Theory, 58, 273-303.

Chamley, C. and Gale, D., 1994, Information Revelation and Strategic Delay in a Model of Investment, Econometrica, 62, 1065-85.

Decamps, J.-P. and Mariotti, T., 2004, Investment Timing and Learning Externalities, Journal of Economic Theory, 118, 80-102.

Grenadier, S.R., 1996, The Strategic Exercise of Options: Development Cascades and Overbuilding in Real Estate Markets, Journal of Finance, 51, 1653-79.

Grenadier, S.R., 1999, Information Revelation Through Option Exercise, Review of Financial Studies, 12, 95-129.

Horstmann, I.J. and Markusen, J.R., 1996, Exploring New Markets: Direct Investment, Contractual Relations and the Multinational Enterprise, International Economic Review, $37,1-19$.

Horstmann, I.J. and Markusen, J.R., 2018, Learning to Sell in New Markets: A Preliminary Analysis of Market Entry by a Multinational Firm, Review of International Economics, $26,1040-52$.

\footnotetext{
${ }^{20}$ A sufficient condition for this is $\Pi^{\mathrm{NP}}>\Pi^{\mathrm{P}}$. An interesting observation is that this asymmetric equilibrium is actually Pareto superior to the symmetric equilibrium because the second mover is strictly better off while the first mover's expected payoff stays the same. This implies that there may exist a collusive asymmetric equilibrium if we allow the firms to transfer payoffs in some binding way.

${ }^{21}$ It is unlikely to attain such coordination because market entry occurs only once and the firms in our setting do not interact repeatedly over time. More generally, in entry games, the identity of potential competitors may not be perfectly known to each other.
} 
Lahiri, S. and Ono, Y., 1988, Helping Minor Firms Reduces Welfare, Economic Journal, 98, 1199-202.

Levin, D. and Peck, J., 2003, To Grab for the Market or to Bide One's Time: A Dynamic Model of Entry, RAND Journal of Economics, 34, 536-56.

Lieberman, M.B. and Montgomery, D.B., 1988, First-Mover Advantages, Strategic Management Journal, 9, 41-58.

Lilien, G.K. and Yoon, E., 1990, The Timing of Competitive Market Entry: An Exploratory Study of New Industry Products, Management Science, 36, 568-85.

Mankiw, N.G. and Whinston, M.D., 1986, Free Entry and Social Efficiency, RAND Journal of Economics, 17, 48-58.

Murto, P. and Välimäki, J., 2011, Learning and Information Aggregation in an Exit Game, Review of Economic Studies, 78, 1426-61.

Pawlina, G. and Kort, P.M., 2006, Real Options in an Asymmetric Oligopoly: Who Benefits from Your Competitive Disadvantage? Journal of Economics and Management Strategy, $15,1-35$.

Rasmusen, E. and Yoon, Y.-R., 2012, First versus Second Mover Advantage with Information Asymmetry about the Profitability of New Markets, Joural of Industrial Economics, 60, 374-405.

Robinson, W.T. and Fornell, C., 1985, Sources of Market Pioneer Advantages in Consumer Goods Industries, Journal of Marketing Research, 22, 305-17.

Shackleton, M.B., Tsekrekos, A.E., and Wojakowski, R., 2004, Strategic Entry and Market Leadership in a Two-Player Real Options Game, Journal of Banking and Finance, 28, 179-201.

Suzumura, K. and Kiyono, K., 1987, Entry Barriers and Economic Welfare, Review of Economic Studies, 54, 157-67.

Trigeorgis, L., 1991, Anticipated Competitive Entry and Early Preemptive Investment in Deferrable Projects, Journal of Business and Economics, 43, 143-56.

Weeds, H., 2002, Strategic Delay in a Real Options Model of R\&D Competition, Review of Economic Studies, 69, 729-47.

Zachary, M.A., Gianiodis, P.T., Payne, G.T., and Markman, G.D., Entry Timing: Enduring Lessons and Future Directions, Journal of Management, 41, 1388-415. 


\section{Appendix}

Proof of Proposition 1. To prove the proposition, we first establish the following facts.

Lemma $3 \lim _{\Delta \rightarrow 0} s \Delta<1$ for any $\phi \geq \bar{p}$.

Proof. Suppose that $\lim _{\Delta \rightarrow 0} s \Delta=1$ for some $(p, q)$. This means that each firm earns $p \frac{\rho \pi}{r}-c$ by entering now. Now suppose that a firm deviates and delays entry until the next instant. In this case, if the other firm does not enter now, the firm knows for sure that the market is bad, and the belief drops to zero, making it optimal never to enter. Taking this into account, the expected profit is

$$
(1-r \Delta)\left(p \frac{\pi}{r}-(p+q(1-\lambda \Delta)) c\right)
$$

which is larger than $p \frac{\pi}{r}-c$ if $\Delta$ is sufficiently small, which is a contradiction.

Lemma $4 s=0$ if $\phi \geq \bar{p}>p$.

Proof. Suppose otherwise, i.e., $s>0$. This is the case if

$$
(1-s \Delta) s^{\prime} \Delta p \rho>(1-p) \lambda c-r\left(p \frac{\pi}{r}-c\right) .
$$

Note that the right-hand side is strictly positive by definition when $\bar{p}>p$, which implies that $\lim _{\Delta \rightarrow 0} s^{\prime} \Delta>0$ and $p>p^{\prime}$. Given this, we can apply the same argument to show that $\lim _{\Delta \rightarrow 0} s_{t} \Delta>0$ for all future periods. Given $\bar{p}>p$, we can take an arbitrarily small $\varepsilon$ and define

$$
N_{\varepsilon}(\Delta):=\max \{k \in \mathbb{N} \mid \varepsilon>k \Delta\}
$$

for some $\varepsilon$. Then, we must have $\lim _{\Delta \rightarrow 0} \Pi_{k=0}^{N_{\varepsilon}(\Delta)}\left(1-s_{t+k \Delta} \Delta\right)=0$. This means that if the other firm does not enter by $t+N_{\varepsilon}(\Delta) \Delta$, the firm's belief will drop almost to zero. By the same argument as in Lemma 3 , it is strictly better to wait until $t+N_{\varepsilon}(\Delta) \Delta$, and hence $s=0$ which is a contradiction.

Lemma 5 For $\phi \geq \bar{p}$

1. $s>0$ if $p \geq \bar{p}$;

2. $\lim _{\Delta \rightarrow 0} s \Delta>0$ such that $\bar{p}>\lim _{\Delta \rightarrow 0} p^{\prime}$ if $p>\bar{p}$, where $p^{\prime}$ indicates the belief at the next instant (in case of no entry). 
Proof. We first show that $s>0$. Suppose that $s=0$. Then $p^{\prime}>p$, and the continuation payoff is weakly lower than

$$
(1-r \Delta)\left(p\left(\frac{\pi}{r}+\rho \Delta\right)-(p+(1-p)(1-\lambda \Delta)) c\right)
$$

which is in turn lower than $p\left(\frac{\pi}{r}+\rho \Delta\right)-c$, the expected payoff of entering now, if $p \geq \bar{p}$.

To prove the second statement, note that

$$
(1-p) \lambda c-r\left(p \frac{\pi}{r}-c\right)<0
$$

so that (5) must be satisfied. As such, it is strictly better to enter now than at the next instant. By Lemma 3, however, a firm, say firm 1, waits for an instant with positive probability. This implies that firm 1's entry decision at the next instant must depend on what firm 2 does now, for otherwise there would be no incentive for firm 1 to wait. The next-period belief must hence be low enough to satisfy $\bar{p}>p^{\prime}$ so that firm 1 enters at the next instant if and only if firm 2 enters now.

Lemma 6 For $\phi \geq \bar{p}, s \in(0, \infty)$ such that $p^{\prime}=\bar{p}$ if $p=\bar{p}$.

Proof. From Lemmas 3 and 4 , we know that a firm must play a mixed strategy if $p \geq \bar{p}$. Suppose first that $s$ is small enough and $p^{\prime}>p=\bar{p}$. Then, $s^{\prime} \Delta>0$ by Lemma 5 , which implies that (5) strictly holds given $p=\bar{p}$. This implies, however, that it is strictly better to enter now than at the next instant, which is a contradiction. Now suppose that $s$ is large enough and $p^{\prime}<\bar{p}$. Then, $s^{\prime}=0$ by Lemma 4 , so that

$$
(1-s \Delta) s^{\prime} \Delta p \rho=(1-p) \lambda c-r\left(p \frac{\pi}{r}-c\right)=0 .
$$

This means that the expected payoff of entering now is equal to that of entering at the next instant regardless of the other firm's action. Given $p^{\prime}<\bar{p}$, however, the optimal strategy at the next instant is to enter if and only if the other firm enters now. Therefore, a firm can earn a higher payoff by waiting for an instant, which is a contradiction. This proves that the belief must be kept at $\bar{p}$ once it reaches this level.

These results show that starting from some $p_{t}<\bar{p}$, no firm enters until $p_{t}$ reaches $\bar{p}$. Once the belief reaches $\bar{p}$, then the firms start randomizing so as to keep $p_{t}=\bar{p}$ as suggested by Lemmas 5 and 6 .

Proof of Proposition 2. We first establish some important properties which must hold in the preemption game. 
Lemma $7 \sigma_{t} \Delta<1$ for any $\phi_{t}<\bar{p}$.

Proof. If $\sigma_{t} \Delta=1$, neither firm can earn monopoly profit. Then, one of the firms can achieve a higher payoff by waiting until the belief reaches $\bar{p}$.

Lemma 8 There exists no $t>0$ such that $\phi_{t}<\bar{p}$ and $\sigma_{t}=\infty$ for all $s \in(0, t)$.

Proof. Note that if $p_{t}$ is close enough to zero, $\sigma_{t}=0$. Suppose that there exists some $t$ such that $\sigma_{s}=\infty$ for all $s \in(0, t)$. However, if $\sigma_{s}=\infty$ for all $s \in\left(0, \frac{t}{2}\right]$, then $p_{\frac{t}{2}} \rightarrow 0$ and hence $\sigma_{\frac{t}{2}} \rightarrow 0$ as $\Delta \rightarrow 0$, which is a contradiction.

Lemma $9 \lim _{\Delta \rightarrow 0} \sigma_{t}<\infty$ for any $\phi_{t}<\bar{p}$ and $t>0$.

Proof. Given some equilibrium strategy $\sigma$, suppose that $\sigma_{t}=\infty$ for some $\phi_{t}<\bar{p}$ and $t>0$. By Lemma 8, there exists some $s \in(0, t)$ such that $\sigma_{s}<\infty$. Without loss of generality, consider $p_{t}$ and $q_{t}$ such that $\sigma_{t-\Delta}<\infty$ with $p_{t-\Delta}$ and $q_{t-\Delta}$ satisfying (1) and (2). Then, if a firm enters at $t-\Delta$, the expected payoff is

$$
p_{t-\Delta} \frac{\pi+\left(1-\sigma_{t-\Delta} \Delta\right)\left(1-e^{-r \delta_{t-\Delta}}\right) \rho}{r}-c .
$$

If it waits and enters at $t$, the expected payoff is

$$
(1-r \Delta) \frac{p_{t-\Delta}}{p_{t}}\left(p_{t} \frac{\pi+\left(1-\sigma_{t} \Delta\right)\left(1-e^{-r \delta_{t}}\right) \rho}{r}-c\right)
$$

which is strictly lower than the payoff of entering at $t-\Delta$. We should thus have $\sigma_{t-\Delta} \Delta=1$, which is a contradiction.

We can now prove the proposition. Since $\phi_{t}$ is strictly increasing, it will reach $\bar{p}$ sooner or later, and the firms enter at some positive rate as shown in Proposition 1. This means that there must be a period in which $\sigma_{t}=0$, followed by a period in which $\sigma_{t} \in(0, \infty)$. To show that $\sigma_{t} \in(0, \infty)$ for $t \in\left(\tau^{*}, \underline{\tau}\right)$, note from Lemma 9 that $\sigma_{t}<\infty$ for $t \in\left(\tau^{*}, \underline{\tau}\right)$. This means that we only need to show that $\sigma_{t}>0$ for all $t \in\left(\tau^{*}, \underline{\tau}\right)$. Suppose that there exists an interval $(\underline{a}, \bar{a})$ such that $\sigma_{t}=0$ for $t \in(\underline{a}, \bar{a})$ but $\sigma_{t}>0$ for $t \in(\underline{a}-\varepsilon, \underline{a}) \cup(\bar{a}, \bar{a}+\varepsilon)$ where $\varepsilon$ is some positive number. This implies that a firm obtains a higher payoff by entering at $\underline{a}$ or at $\bar{a}$ than at any time in $(\underline{a}, \bar{a})$. Given that $\sigma_{t}=0$ for $t \in(\underline{a}, \bar{a})$, by entering at $t+d t$ instead of entering at $t$, the firm's expected payoff increases by

$$
(r+\lambda) c-p_{t}\left(\pi+\left(1+e^{-r \delta_{t}}\right) \rho+\lambda c\right),
$$


which is decreasing in $t$. This means that the expected payoff is concave in $(\underline{a}, \bar{a})$, and hence we cannot have the payoff maximized at $\underline{a}$ and $\bar{a}$ in this interval. Therefore, $\sigma_{t} \in(0, \infty)$ for $t \in\left(\tau^{*}, \underline{\tau}\right)$.

Finally, note that when $\phi_{t}=\bar{p}$, it is strictly optimal for a firm to wait until $p_{t}$ reaches $\bar{p}$. Therefore, shortly before $\phi_{t}$ reaches $\bar{p}$, it is still optimal to wait, meaning that $\phi_{\underline{\tau}}<\bar{p}$.

Proof of Lemma 2. Observe that $\tau^{\mathrm{P}}$ is the solution to

$$
\max _{t} \frac{p_{0}(\pi+\rho-r c)}{r} e^{-r t}-\frac{p_{0} \rho}{r} e^{-r\left(\tau^{\mathrm{NP}}-t\right)}-\left(1-p_{0}\right) c e^{-(\lambda+r) t} .
$$

The first-order condition is then given by

$$
\mu(t):=-p_{0}(\pi+\rho-r c)-p_{0} \rho e^{-r\left(\tau^{\mathrm{NP}}-2 t\right)}+\left(1-p_{0}\right)(\lambda+r) c e^{-\lambda t} .
$$

It is straightforward to verify that $\mu$ is strictly decreasing in $t$ for $t \in\left[0, \frac{\tau^{\mathrm{NP}}}{2}\right]$, meaning that there exists at most one $\hat{\tau}$ such that $\mu(\hat{\tau})=0$. In the limit, the optimal timing of pioneering entry, denoted by $\tau^{\mathrm{P}}$, is given by

$$
\tau^{\mathrm{P}}= \begin{cases}0 & \text { if } 0 \geq \mu(0) \\ \hat{\tau} & \text { if } \mu(0)>0>\mu\left(\frac{\tau^{\mathrm{NP}}}{2}\right), \\ \frac{\tau^{\mathrm{NP}}}{2} & \text { if } \mu\left(\frac{\tau^{\mathrm{NP}}}{2}\right) \geq 0\end{cases}
$$

where $\hat{\tau}$ solves $\mu(\hat{\tau})=0$.

Proof of Proposition 3. The sufficiency is obvious. If $\Pi^{\mathrm{P}}>\Pi^{\mathrm{NP}}$, there is an incentive for a firm to enter the market when $\bar{p}>\phi_{t}$. There must be an equilibrium with pioneering entry.

To show the necessity, we define $\Pi^{\mathrm{NP}}$ as a function of the initial prior $p_{0}$. We can then establish the following fact.

Lemma 10 If pioneering entry occurs,

$$
p_{\tau^{*}} \frac{\pi+\left(1-e^{-r \delta_{\tau^{*}}}\right) \rho}{r}-c>\Pi^{\mathrm{NP}}\left(p_{\tau^{*}}\right) .
$$

Proof. Suppose that pioneering entry occurs. Then, by Proposition $2, \sigma_{t}<\infty$ for $t>0$, and the expected payoff of entering at $t$ is

$$
p_{t} \frac{\pi+\left(1-e^{-r \delta_{t}}\right) \rho}{r}-c .
$$

As discussed in the proof of Proposition 2, a firm must be indifferent between entering at $\underline{\tau}$ and at $\bar{\tau}$, at which the belief reaches $\bar{p}$, i.e.,

$$
p_{\underline{\tau}} \frac{\pi+\left(1-e^{-r \delta_{\underline{\tau}}}\right) \rho}{r}-c=\Pi^{\mathrm{NP}}\left(p_{\underline{\tau}}\right) .
$$


Moreover, at any $t \in\left(\tau^{*}, \underline{\tau}\right)$, a firm must be indifferent between entering and waiting. This means that a firm's payoff of entering at $\tau^{*}$ must equal to that of becoming the second mover (observes the other firm's entry and then waits until the belief reaches $\bar{p}$ ), i.e., in either contingency, the firm's payoff is

$$
p_{\tau^{*}} \frac{\pi+\left(1-e^{-r \delta_{\tau^{*}}}\right) \rho}{r}-c .
$$

Since $\Pi^{N P}$ is the payoff when a firm does not gain any information from the rival, it is necessarily lower than the payoff of becoming the second mover, which proves the lemma.

Given this, since $\Pi^{\mathrm{NP}}\left(p_{0}\right)=e^{-r \tau^{*}} \frac{p_{0}}{p_{\tau^{*}}} \Pi^{\mathrm{NP}}\left(p_{\tau^{*}}\right)$ and

$$
\Pi^{\mathrm{P}}\left(p_{0}\right) \geq e^{-r \tau^{*}} \frac{p_{0}}{p_{\tau^{*}}}\left(p_{\tau^{*}} \frac{\pi+\left(1-e^{-r \delta_{\tau^{*}}}\right) \rho}{r}-c\right),
$$

we can show that $\Pi^{\mathrm{P}}\left(p_{0}\right)>\Pi^{\mathrm{NP}}\left(p_{0}\right)$.

Proof of Proposition 4. Note that $\Pi^{\mathrm{NP}}$ and $\Pi^{\mathrm{P}}$ are given by

$$
\begin{aligned}
& \Pi^{\mathrm{NP}}\left(p_{0}\right)=e^{-r \tau^{\mathrm{NP}}}\left(p_{0} \frac{\pi}{r}-\left(p_{0}+\left(1-p_{0}\right) e^{-\lambda \tau^{\mathrm{NP}}}\right) c\right), \\
& \Pi^{\mathrm{P}}\left(p_{0}\right)=e^{-r \tau^{\mathrm{P}}}\left(p_{0} \frac{\pi+\left(1-e^{-r\left(\tau^{\mathrm{NP}}-2 \tau^{\mathrm{P}}\right)}\right) \rho}{r}-\left(p_{0}+\left(1-p_{0}\right) e^{-\lambda \tau^{\mathrm{P}}}\right) c\right) .
\end{aligned}
$$

It is clear that (14) can only increase with $\rho$ while (13) is independent of it. Therefore, there must be a threshold $\hat{\rho}$ such that the condition for pioneering entry is satisfied if and only if $\rho>\hat{\rho}$.

For the effect of $p_{0}$, define $p_{0}^{\prime \prime}:=\frac{p_{0}^{\prime}}{p_{0}^{\prime}+\left(1-p_{0}^{\prime}\right) e^{-\lambda \Delta}}$ for a given $p_{0}^{\prime}<\bar{p}$ where $\Delta$ is assumed to be arbitrarily small. Also, we write $\tau^{\mathrm{NP}}$ and $\tau^{\mathrm{P}}$ both as functions of $p_{0}$. Since

$$
\Pi^{\mathrm{NP}}\left(p_{0}\right)=e^{-r \tau^{\mathrm{NP}}\left(p_{0}\right)} \frac{p_{0}}{\bar{p}}\left(\bar{p} \frac{\pi}{r}-c\right),
$$

we have

$$
\Pi^{\mathrm{NP}}\left(p_{0}^{\prime \prime}\right)=\frac{e^{-r \tau^{\mathrm{NP}}\left(p_{0}^{\prime \prime}\right)} p_{0}^{\prime \prime}}{e^{-r \tau^{\mathrm{NP}}\left(p_{0}^{\prime}\right)} p_{0}^{\prime}} \Pi^{\mathrm{NP}}\left(p_{0}^{\prime}\right)=e^{r \Delta} \frac{p_{0}^{\prime \prime}}{p_{0}^{\prime}} \Pi^{\mathrm{NP}}\left(p_{0}^{\prime}\right),
$$

for any $\bar{p}>p_{0}^{\prime \prime}>p_{0}^{\prime}$.

As for $\Pi^{\mathrm{P}}$, observe first that by Lemma 2 ,

$$
\begin{aligned}
\lim _{p_{0} \uparrow \bar{p}} \mu(0) & =-p_{0}(\pi+\rho-r c)-p_{0} \rho e^{-r \tau^{\mathrm{NP}}}+\left(1-p_{0}\right)(\lambda+r) c \\
& =-\frac{(\lambda+r) c}{\pi+\lambda c}(\pi+\rho-r c)-\frac{(\lambda+r) c}{\pi+\lambda c} \rho e^{-r \tau^{\mathrm{NP}}}+\frac{\pi-r c}{\pi+\lambda c}(\lambda+r) c<0,
\end{aligned}
$$


suggesting that there exists some threshold $\tilde{p}<\bar{p}$ such that $\tau^{\mathrm{P}}=0$ if and only if $p_{0} \in[\tilde{p}, \bar{p})$. Also, define $p^{+}:=\frac{p_{0}^{\prime}}{p_{0}^{\prime}+\left(1-p_{0}^{\prime}\right) e^{-\lambda \tau^{\mathrm{P}}\left(p_{0}^{\prime}\right)}}$.

First consider the case where $p_{0}<\tilde{p}$, or alternatively $p^{+} \geq p_{0}^{\prime \prime}$ and $\tau^{\mathrm{P}}\left(p_{0}^{\prime}\right) \geq \Delta$. We then have

$$
\begin{aligned}
\Pi^{\mathrm{P}}\left(p_{0}^{\prime}\right) & =e^{-r \tau^{\mathrm{P}}\left(p_{0}^{\prime}\right)}\left(p_{0}^{\prime} \frac{\pi+\left(1-e^{-r\left(\tau^{\mathrm{NP}}\left(p_{0}^{\prime}\right)-2 \tau^{\mathrm{P}}\left(p_{0}^{\prime}\right)\right)}\right) \rho}{r}-\left(p_{0}^{\prime}+\left(1-p_{0}^{\prime}\right) e^{-\lambda \tau^{\mathrm{P}}\left(p_{0}^{\prime}\right)}\right) c\right) \\
& =e^{-r \tau^{\mathrm{P}\left(\mathrm{p}_{0}^{\prime}\right)}} \frac{p_{0}^{\prime}}{p^{+}}\left(p^{+} \frac{\pi+\left(1-e^{-r\left(\tau^{\mathrm{NP}}\left(p_{0}^{\prime}\right)-2 \tau^{\mathrm{P}}\left(p_{0}^{\prime}\right)\right)}\right) \rho}{r}-c\right) .
\end{aligned}
$$

Similarly,

$$
\begin{aligned}
\Pi^{\mathrm{P}}\left(p_{0}^{\prime \prime}\right) & =e^{-r \tau^{\mathrm{P}}\left(p_{0}^{\prime \prime}\right)}\left(p_{0}^{\prime \prime} \frac{\pi+\left(1-e^{-r\left(\tau^{\mathrm{NP}}\left(p_{0}^{\prime \prime}\right)-2 \tau^{\mathrm{P}}\left(p_{0}^{\prime \prime}\right)\right)}\right) \rho}{r}-\left(p_{0}^{\prime \prime}+\left(1-p_{0}^{\prime \prime}\right) e^{-\lambda \tau^{\mathrm{P}}\left(p_{0}^{\prime \prime}\right)}\right) c\right) \\
& >e^{-r\left(\tau^{\mathrm{P}}\left(p_{0}^{\prime}\right)-\Delta\right)} \frac{p_{0}^{\prime \prime}}{p^{+}}\left(p^{+} \frac{\pi+\left(1-e^{-r\left(\tau^{\mathrm{NP}}\left(p_{0}^{\prime \prime}\right)-2\left(\tau^{\mathrm{P}}\left(p_{0}^{\prime}\right)-\Delta\right)\right.}\right) \rho}{r}-c\right) \\
& >e^{-r\left(\tau^{\mathrm{P}}\left(p_{0}^{\prime}\right)-\Delta\right)} \frac{p_{0}^{\prime \prime}}{p^{+}}\left(p^{+} \frac{\pi+\left(1-e^{-r\left(\tau^{\mathrm{NP}}\left(p_{0}^{\prime}\right)-2 \tau^{\mathrm{P}}\left(p_{0}^{\prime}\right)\right)}\right) \rho}{r}-c\right) \\
& =e^{r \Delta} \frac{p_{0}^{\prime \prime}}{p_{0}^{\prime}} \Pi^{\mathrm{P}}\left(p_{0}^{\prime}\right) .
\end{aligned}
$$

Here, the second line shows the payoff when the firm enters at $\tau^{\mathrm{P}}\left(p_{0}^{\prime}\right)-\Delta$ at which point the belief reaches $p^{+}$. From the second line to the third, we use the fact that

$$
\tau^{\mathrm{NP}}\left(p^{\prime \prime}\right)+2 \Delta>\tau^{\mathrm{NP}}\left(p^{\prime \prime}\right)+\Delta=\tau^{\mathrm{NP}}\left(p_{0}^{\prime}\right)
$$

Next, consider the case where $p_{0}^{\prime} \geq \tilde{p}$, or alternatively $p_{0}^{\prime \prime}>p^{+}$and $\tau^{\mathrm{P}}\left(p_{0}^{\prime}\right)=0$. We then have

$$
\Pi^{\mathrm{P}}\left(p_{0}^{\prime}\right)=p_{0}^{\prime} \frac{\pi+\left(1-e^{-r \tau^{\mathrm{NP}}\left(p_{0}^{\prime}\right)}\right) \rho}{r}-c>e^{-r \Delta} \frac{p_{0}^{\prime}}{p_{0}^{\prime \prime}} \Pi^{\mathrm{P}}\left(p_{0}^{\prime \prime}\right),
$$

and

$$
\Pi^{\mathrm{P}}\left(p_{0}^{\prime \prime}\right)=p_{0}^{\prime \prime} \frac{\pi+\left(1-e^{-r \tau^{\mathrm{NP}}\left(p_{0}^{\prime \prime}\right)}\right) \rho}{r}-c
$$

It follows from above that if $\pi^{\mathrm{NP}}\left(p_{0}^{\prime}\right)=\pi^{\mathrm{P}}\left(p_{0}^{\prime}\right)$, then $\pi^{\mathrm{NP}}\left(p_{0}^{\prime \prime}\right)<\pi^{\mathrm{P}}\left(p_{0}^{\prime \prime}\right)$ for $\tilde{p}>p_{0}^{\prime}$ and $\pi^{\mathrm{NP}}\left(p_{0}^{\prime \prime}\right)>\pi^{\mathrm{P}}\left(p_{0}^{\prime \prime}\right)$ for $p_{0} \geq \tilde{p}$. This suggests that $\Pi^{\mathrm{NP}}$ and $\Pi^{\mathrm{P}}$ intersect at most twice for $p_{0} \in(0, \bar{p}]$. Note also that $\bar{p}>\tilde{p}$ and $\Pi^{\mathrm{NP}}(\bar{p})=\Pi^{\mathrm{P}}(\bar{p})$. This proves that there is a threshold $\hat{p} \in(0, \bar{p})$ such that the condition for pioneering entry is satisfied if and only if $p_{0} \in(\hat{p}, \bar{p})$.

Proof of Proposition 5. For the first inequality, observe that $\lim _{t \downarrow \frac{\tau \mathrm{NP}}{2}} \hat{\Pi}_{0}^{\prime}(t)>0$ by definition. This implies that if $\tau^{\mathrm{P}}=\frac{\tau^{\mathrm{NP}}}{2}$, then $\Pi^{\mathrm{NP}}>\Pi^{\mathrm{P}}$.

For the second inequality, we make use of the following results. 
Lemma 11 If pioneering entry occurs in equilibrium, then $\tau^{\mathrm{P}} \geq \tau^{*}$.

Proof. If $\tau>\hat{\tau}$, a firm can deviate by entering at $t \in[\hat{\tau}, \tau)$ and obtain a higher payoff.

Lemma 12 If pioneering entry occurs in equilibrium,

$$
p_{0}\left(\pi+\left(1+e^{-r\left(\tau^{\mathrm{NP}}-2 \tau^{\mathrm{P}}\right)}\right) \rho-r c\right) \geq\left(1-p_{0}\right) e^{-\lambda \tau^{\mathrm{P}}}(r+\lambda) c .
$$

Proof. By the definition of $\tau^{\mathrm{P}}$, if

$$
\left(1-p_{0}\right) e^{-\lambda \tau^{\mathrm{P}}}(r+\lambda) c>p_{0}\left(\pi+\left(1+e^{-r\left(\tau^{\mathrm{NP}}-2 \tau^{\mathrm{P}}\right)}\right) \rho-r c\right)
$$

then $\tau^{\mathrm{P}}=\frac{\tau^{\mathrm{NP}}}{2}$, so that the first mover cannot earn any monopoly profit. This means, however, that $\Pi^{\mathrm{NP}}>\Pi^{\mathrm{P}}$, i.e.,

$$
e^{-r \tau^{\mathrm{NP}}}\left(p_{0} \frac{\pi}{r}-\left(p_{0}+\left(1-p_{0}\right) e^{-\lambda \tau^{\mathrm{NP}}}\right) c\right)>e^{-r \tau^{\mathrm{P}}}\left(p_{0} \frac{\pi}{r}-\left(p_{0}+\left(1-p_{0}\right) e^{-\lambda \tau^{\mathrm{P}}}\right) c\right),
$$

which suggests no pioneering entry in equilibrium, a contradiction.

We are now ready to prove the proposition. If a firm, say firm 1 , enters at $\tau^{*}$, the expected payoff is

$$
\Pi_{\tau^{*}}:=p_{\tau^{*}} \frac{\pi+\left(1-\sigma_{\tau^{*}} \Delta\right)\left(1-e^{-r \delta_{\tau^{*}}}\right) \rho}{r}-c .
$$

If firm 1 waits for a period, on the other hand, firm 2 enters with probability $\sigma_{\tau^{*}} \Delta$, in which case firm 1 waits until $\tau^{*}+\delta_{\tau^{*}}$ when $p_{t}$ reaches $\bar{p}$; with the remaining probability, firm 2 does not enter at $\tau^{*}$, and firm 1 enters at $\tau^{*}+\Delta$ if it observes no signal. Letting $x_{t}:=\left(1-\sigma_{t} \Delta\right)\left(p_{t}+q_{t} e^{-\lambda \Delta}\right)+\left(1-p_{t}-q_{t}\right) e^{-\lambda \Delta}$, the expected payoff of waiting for a period is given by

$\sigma_{\tau^{*}} \Delta e^{-r \delta_{\tau^{*}}} \frac{p_{\tau^{*}}}{\bar{p}}\left(\bar{p} \frac{\pi}{r}-c\right)+(1-r \Delta)\left(\left(1-\sigma_{\tau^{*}} \Delta\right) p_{\tau} \frac{\pi+\left(1-\sigma_{\tau^{*}+\Delta} \Delta\right)\left(1-e^{-r\left(\delta_{\tau^{*}}-2 \Delta\right)}\right) \rho}{r}-x_{\tau^{*}} c\right)$.

Now suppose that $\tau^{*}=\tau^{\mathrm{P}}>0$. Since $\lim _{\Delta \rightarrow 0} \sigma_{\tau^{*}}<\infty$ by Lemma 9 and $\lim _{\Delta \rightarrow 0} \sigma_{\tau^{*}+\Delta}=$ $\sigma_{\tau^{*}}$ by the continuity of $\sigma_{t},(17)$ is reduced to

$$
(1-r \Delta) \Pi_{\tau^{*}}-\sigma_{\tau^{*}} \Delta \Lambda-p_{\tau^{*}} \frac{2 r e^{-r \delta_{\tau^{*}}} \rho}{r} \Delta-\left(1-p_{\tau^{*}}\right) \lambda c \Delta,
$$

where

$$
\Lambda:=\Pi_{\tau^{*}}+\left(1-p_{\tau^{*}}-q_{\tau^{*}}\right) c-e^{-r \delta_{\tau^{*}}} \frac{p_{\tau^{*}}}{\bar{p}}\left(\bar{p} \frac{\pi}{r}-c\right) .
$$


From (16) and (18), the firm is indifferent if

$$
\sigma_{\tau^{*}} \Lambda+p_{\tau^{*}}\left(\pi+\left(1+e^{-r \delta_{\tau^{*}}}\right) \rho+\lambda c\right)=(r+\lambda) c
$$

Given this, we next show that $\Lambda>0$ if $\tau^{*}=\tau^{\mathrm{P}}$. Note that

$$
\Pi_{\tau^{*}}=e^{r \tau^{*}} \frac{p_{\tau^{*}}}{p_{0}} \Pi^{\mathrm{P}}, e^{-r\left(\tau^{\mathrm{NP}}-\tau^{*}\right)} \frac{p_{\tau^{*}}}{\bar{p}}\left(\bar{p} \frac{\pi}{r}-c\right)=e^{r \tau^{*}} \frac{p_{\tau^{*}}}{p_{0}} \Pi^{\mathrm{NP}} .
$$

It follows from these that

$$
\Lambda=e^{r \tau^{*}} \frac{p_{\tau^{*}}}{p_{0}}\left(\Pi^{\mathrm{P}}-\Pi^{\mathrm{NP}}\right)+\left(1-p_{\tau^{*}}-q_{\tau^{*}}\right) c-\left(e^{-r \delta_{\tau^{*}}}-e^{-r\left(\tau^{\mathrm{NP}}-\tau^{*}\right)}\right) \frac{p_{\tau^{*}}}{\bar{p}}\left(\bar{p} \frac{\pi}{r}-c\right) .
$$

Since $\Pi^{\mathrm{P}}>\Pi^{\mathrm{NP}}$ when pioneering entry occurs, we obtain

$$
\left(1-p_{\tau^{*}}-q_{\tau^{*}}\right) c>\left(e^{-r \delta_{\tau^{*}}}-e^{-r\left(\tau^{\mathrm{NP}}-\tau^{*}\right)}\right) \frac{p_{\tau^{*}}}{\bar{p}}\left(\bar{p} \frac{\pi}{r}-c\right) \Rightarrow \Lambda>0 .
$$

Given that $q_{\tau^{*}}=\left(1-p_{\tau^{*}}\right) e^{-\lambda \tau^{*}}$ and $\delta_{t}=\tau^{\mathrm{NP}}-2 t$, this condition can be written as

$$
\left(1-p_{\tau^{*}}\right)\left(1-e^{-\lambda \tau^{*}}\right) c>e^{-r\left(\tau^{\mathrm{NP}}-2 \tau^{*}\right)}\left(1-e^{-r \tau^{*}}\right) \frac{p_{\tau^{*}}}{\bar{p}}\left(\bar{p} \frac{\pi}{r}-c\right) .
$$

To show that (20) holds, by the definition of $\bar{p}$, we have

$$
(1-\bar{p}) \lambda c=r\left(\bar{p} \frac{\pi}{r}-c\right) \Leftrightarrow\left(1-p_{\tau^{*}}\right) e^{-\lambda \delta_{\tau^{*}}} \lambda c=r \frac{p_{\tau^{*}}}{\bar{p}}\left(\bar{p} \frac{\pi}{r}-c\right) .
$$

Observe that for $t \leq \tau^{*}<\delta_{\tau^{*}}$,

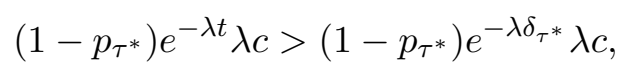

and

$$
r \frac{p_{\tau^{*}}}{\bar{p}}\left(\bar{p} \frac{\pi}{r}-c\right)>e^{-r\left(\tau^{\mathrm{NP}}-2 \tau^{*}\right)} r \frac{p_{\tau^{*}}}{\bar{p}}\left(\bar{p} \frac{\pi}{r}-c\right) e^{-r t}
$$

We hence obtain

$$
\int_{0}^{\tau}\left(1-p_{\tau^{*}}\right) e^{-\lambda t} \lambda c d t>\int_{0}^{\tau} e^{-r\left(\tau^{\mathrm{NP}}-2 \tau^{*}\right)} r \frac{p_{\tau^{*}}}{\bar{p}}\left(\bar{p} \frac{\pi}{r}-c\right) e^{-r t} d t
$$

which can be written as

$$
\left(1-p_{\tau^{*}}\right)\left(1-e^{-\lambda \tau^{*}}\right) c>e^{-r\left(\tau^{\mathrm{NP}}-2 \tau^{*}\right)}\left(1-e^{-r \tau^{*}}\right) \frac{p_{\tau^{*}}}{\bar{p}}\left(\bar{p} \frac{\pi}{r}-c\right) .
$$

This proves $\Lambda>0$.

Now suppose that $\sigma_{\tau^{*}}=0$. Then, (19) holds if

$$
p_{\tau^{*}}\left(\pi+\left(1+e^{-r \delta_{\tau^{*}}}\right) \rho+\lambda c\right)=(r+\lambda) c .
$$


If this is the case, since $\sigma_{t}$ is continuous, there exist $\varepsilon>0$ such that for $t \in(\tau, \tau+\varepsilon), \Lambda_{t}>0$ and $\sigma_{t}$ is so close to zero that

$$
p_{t}\left(\pi+\left(1+e^{-r \delta_{t}}\right) \rho+\lambda c\right)>(r+\lambda) c .
$$

We then have

$$
\sigma_{t} \Lambda+p_{t}\left(\pi+\left(1+e^{-r \delta_{t}}\right) \rho+\lambda c\right)>(r+\lambda) c
$$

meaning that it is strictly better to enter at $t$ than at $t+\Delta$, which is a contradiction. Therefore, $\sigma_{\tau^{*}}>0$.

Finally, if $\tau^{*}=\tau^{\mathrm{P}}$,

$$
p_{\tau^{*}}\left(\pi+\left(1+e^{-r \delta_{\tau^{*}}}\right) \rho+\lambda c\right) \geq(r+\lambda) c,
$$

by Lemma 12. Moreover, since $\Lambda>0$ and $\sigma_{\tau^{*}}>0$, we have

$$
\sigma_{\tau^{*}} \Lambda+p_{\tau^{*}}\left(\pi+\left(1+e^{-r \delta_{\tau^{*}}}\right) \rho+\lambda c\right)>(r+\lambda) c .
$$

This implies $\tau^{*} \neq \tau^{\mathrm{P}}$ by (19) and Lemma 11 further implies $\tau^{\mathrm{P}}>\tau^{*}$.

Proof of Proposition 6. By Lemma 10,

$$
e^{-r \tau^{*}} \frac{p_{0}}{p_{\tau^{*}}}\left(p_{\tau^{*}} \frac{\pi+\left(1-e^{-r \delta_{\tau^{*}}}\right) \rho}{r}-c\right)>\Pi^{\mathrm{NP}} .
$$

By Lemma $11, \tau^{*}=\tau^{\mathrm{P}}$ if $\tau^{\mathrm{P}}=0$. The equilibrium payoff in this case is

$$
p_{0} \frac{\pi+\left(1-\lim _{\Delta \rightarrow 0} \sigma_{0} \Delta\right)\left(1-e^{-r \tau^{\mathrm{NP}}}\right) \rho}{r}-c \leq \Pi^{\mathrm{P}}=p_{0} \frac{\pi+\left(1-e^{-r \tau^{\mathrm{NP}}}\right) \rho}{r}-c .
$$

If $\tau^{\mathrm{P}}>0$, on the other hand, it is clear that the equilibrium payoff is strictly lower than $\Pi^{\mathrm{P}}$.

Proof of Proposition 7. (i) Note that $\tau^{\mathrm{NP}}$ must solve

$$
p_{0}(\pi-r c)=\left(1-p_{0}\right)(\lambda+r) c e^{-\lambda \tau^{\mathrm{NP}}} .
$$

The only difference from (8) is that the left-hand side is $p_{0}(\pi-r c)$ instead of $p_{0}(\pi-\rho-r c)$, which implies that $T^{* *}>\tau^{\mathrm{NP}}$.

(ii) Since $\frac{T^{* *}}{2}>\frac{\tau^{\mathrm{NP}}}{2} \geq \tau^{\mathrm{P}}, \tau_{1}^{* *}>\tau^{\mathrm{P}}$ holds if $\tau_{1}^{* *} \geq \frac{T^{* *}}{2}$. This means that we can focus on $\tau_{1}^{* *}<\frac{T^{* *}}{2}$. In this case, $\tau_{1}^{* *}$ must solve (10), which implies that

$$
e^{-\lambda \tau_{1}^{* *}}<\frac{p_{0}(\pi+\rho-r c)}{\left(1-p_{0}\right)(\lambda+r) c} .
$$


On the other hand, if $\tau^{\mathrm{P}}>0$, it must satisfy

$$
p_{0}(\pi+\rho-r c)=\left(1-p_{0}\right)(\lambda+r) c e^{-\lambda \tau^{\mathrm{P}}}-p_{0} \rho e^{-r\left(\tau^{\mathrm{NP}}-2 \tau^{\mathrm{P}}\right)} .
$$

As this implies that

$$
e^{-\lambda \tau^{\mathrm{P}}}>\frac{p_{0}(\pi+\rho-r c)}{\left(1-p_{0}\right)(\lambda+r) c}
$$

we have $\tau_{1}^{* *}>\tau^{\mathrm{P}}$.

(iii) Plugging $\tau_{1}=\tau^{\mathrm{NP}}$ into (11), the right-hand side becomes

$$
\left(1-p_{0}\right)\left((\lambda+r)+(2 \lambda+r) \frac{p_{0}}{1-p_{0}} \frac{\pi-r c}{(\lambda+r) c}\right) c e^{-\lambda \tau^{\mathrm{NP}}} .
$$

If $\frac{p_{0}(\pi-r c)}{\left(1-p_{0}\right)(\lambda+r) c}<\frac{\lambda+r}{2 \lambda+r}$, the right-hand side is smaller than $2\left(1-p_{0}\right)(\lambda+r) c e^{-\lambda \tau^{\mathrm{NP}}}=2 p_{0}(\pi-r c)$, and thus

$$
2 p_{0}(\pi-r c)>\left(1-p_{0}\right)\left((\lambda+r)+(2 \lambda+r) e^{-\lambda \tau_{1}}\right) c e^{-\lambda \tau_{1}},
$$

for all $\tau_{1} \geq \tau^{\mathrm{NP}}$. Therefore, $\tau_{1}^{* *}<\tau^{\mathrm{NP}}$.

We next show that $\tau_{1}^{* *}$ can be larger than $\tau^{\mathrm{NP}}$ if $\frac{p_{0}(\pi-r c)}{\left(1-p_{0}\right)(\lambda+r) c}>\frac{\lambda+r}{2 \lambda+r}$. Suppose that $\rho=0$. Then $T^{* *}=\tau^{\mathrm{NP}}$, so the right-hand side of (10) is larger than the left-hand side for $\tau_{1} \in\left[0, \frac{T^{* *}}{2}\right)$. Given that $\frac{p_{0}(\pi-r c)}{\left(1-p_{0}\right)(\lambda+r) c}>\frac{\lambda+r}{2 \lambda+r}$,

$$
2 p_{0}(\pi-r c)<\left(1-p_{0}\right)\left((\lambda+r)+(2 \lambda+r) e^{-\lambda \tau_{1}}\right) c e^{-\lambda \tau_{1}},
$$

for $\tau_{1}^{* *} \in\left[\frac{T^{* *}}{2}, \tau^{\mathrm{NP}}\right]$. Therefore, $\tau_{1}^{* *}>\tau^{\mathrm{NP}}$. The same argument applies as long as $\rho$ is close enough to 0 . 


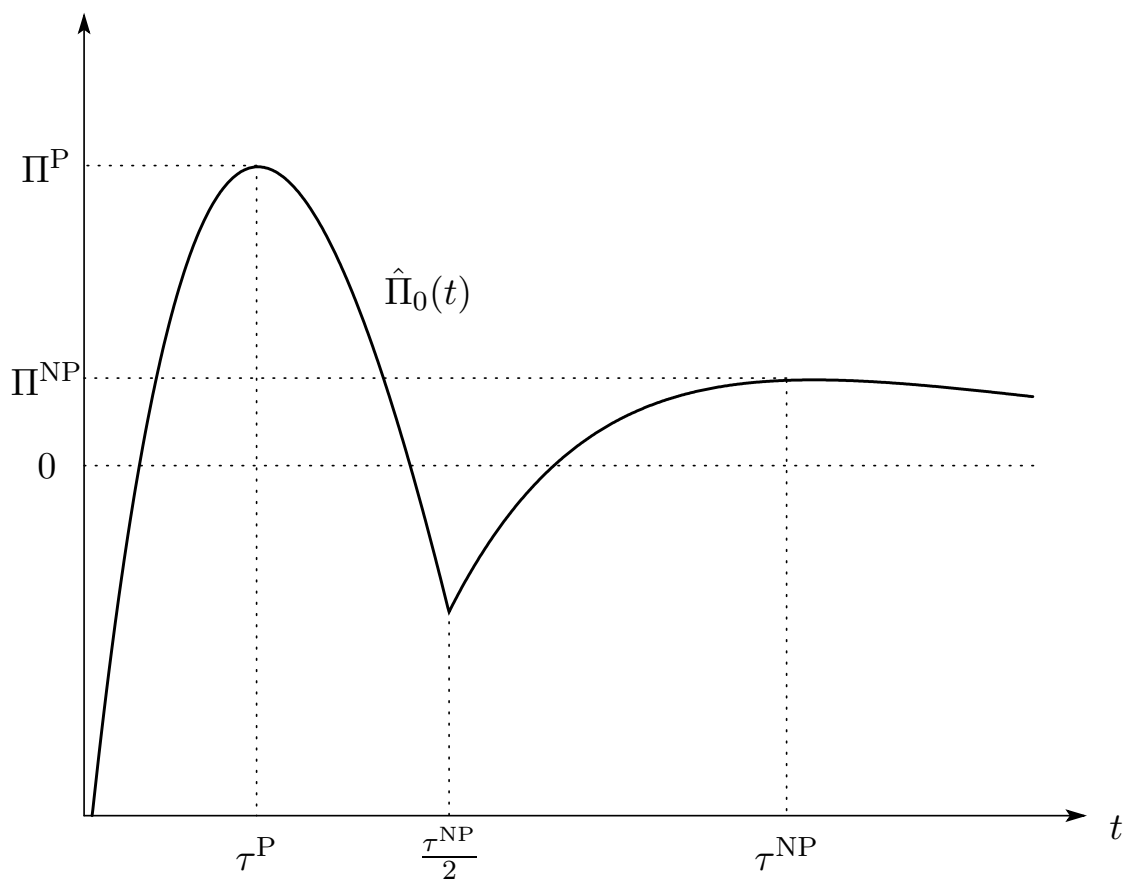

Figure 1: The emergence of a market pioneer $\left(\lambda=0.1, r=0.1, p_{0}=0.3, c=3, \pi=0.5, \rho=0.5\right)$

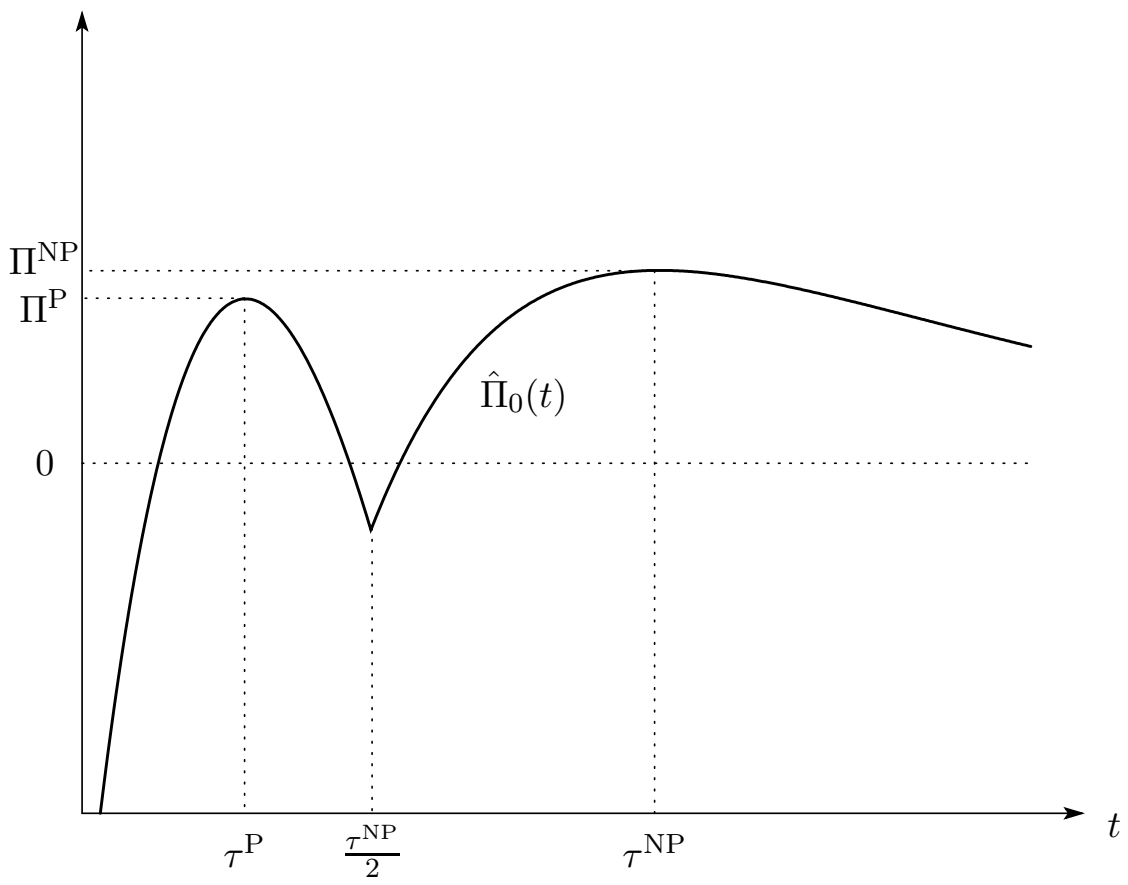

Figure 2: No market pioneer

$$
\left(\lambda=0.1, r=0.1, p_{0}=0.3, c=3, \pi=0.6, \rho=0.4\right)
$$

